Check for updates

Cite this: J. Mater. Chem. B, 2020, 8, 9428

Received 2nd June 2020, Accepted 17th August 2020

DOI: $10.1039 / \mathrm{dOtb01398f}$

rsc.li/materials-b

\title{
Promising nanomaterials in the fight against malaria
}

\author{
Livia Neves Borgheti-Cardoso, (D) $\dagger^{\text {abc }}$ María San Anselmo, (D) $\dagger^{d}$ Elena Lantero, (D) abc \\ Alexandre Lancelot, (iD d José Luis Serrano, (iD d Silvia Hernández-Ainsa, (iD de \\ Xavier Fernàndez-Busquets (iD *abc and Teresa Sierra (iD *d
}

\begin{abstract}
For more than one hundred years, several treatments against malaria have been proposed but they have systematically failed, mainly due to the occurrence of drug resistance in part resulting from the exposure of the parasite to low drug doses. Several factors are behind this problem, including (i) the formidable barrier imposed by the Plasmodium life cycle with intracellular localization of parasites in hepatocytes and red blood cells, (ii) the adverse fluidic conditions encountered in the blood circulation that affect the interaction of molecular components with target cells, and (iii) the unfavorable physicochemical characteristics of most antimalarial drugs, which have an amphiphilic character and can be widely distributed into body tissues after administration and rapidly metabolized in the liver. To surpass these drawbacks, rather than focusing all efforts on discovering new drugs whose efficacy is quickly decreased by the parasite's evolution of resistance, the development of effective drug delivery carriers is a promising strategy. Nanomaterials have been investigated for their capacity to effectively deliver antimalarial drugs at local doses sufficiently high to kill the parasites and avoid drug resistance evolution, while maintaining a low overall dose to prevent undesirable toxic side effects. In recent years, several nanostructured systems such as liposomes, polymeric nanoparticles or dendrimers have been shown to be capable of improving the efficacy of antimalarial therapies. In this respect, nanomaterials are a promising drug delivery vehicle and can be used in therapeutic strategies designed to fight the parasite both in humans and in the mosquito vector of the disease. The chemical analyses of these nanomaterials are essential for the proposal and development of effective anti-malaria therapies. This review is intended to analyze the application of nanomaterials to improve the drug efficacy on different stages of the malaria parasites in both the human and mosquito hosts.
\end{abstract}

\section{Introduction}

As defined by the World Health Organization (WHO), malaria is one of the infectious diseases of poverty (DoP) of global health importance in tropical and subtropical regions. ${ }^{1}$ In $2018,93 \%$ of the malaria cases occurred in the WHO African Region, followed by $3.4 \%$ in the South-East Asian Region, and $2.1 \%$ in the Eastern

\footnotetext{
${ }^{a}$ Nanomalaria Group, Institute for Bioengineering of Catalonia (IBEC), The Barcelona Institute of Science and Technology, Baldiri Reixac 10-12, ES-08028 Barcelona, Spain

${ }^{b}$ Barcelona Institute for Global Health (ISGlobal, Hospital Clinic-Universitat de Barcelona), Rosselló 149-153, ES-08036 Barcelona, Spain

${ }^{c}$ Nanoscience and Nanotechnology Institute (IN2UB), University of Barcelona, Marti i Franquès 1, ES-08028 Barcelona, Spain.

E-mail: xfernandez_busquets@ub.edu

${ }^{d}$ Instituto de Nanociencia y Materiales de Aragón (INMA), Dep. Química Orgánica-Facultad de Ciencias, CSIC-Universidad de Zaragoza, 50009 Zaragoza, Spain. E-mail: tsierra@unizar.es, tsierra@ctq.csic.es ${ }^{e}$ ARAID Foundation, Government of Aragón, Zaragoza 50018, Spain

$\dagger$ These authors contributed equally.
}

Mediterranean Region. ${ }^{2}$ Although the number of infected patients and deaths caused by malaria has decreased from 251 million cases and 585000 deaths in 2010 to 228 million cases and 405000 deaths in $2018,{ }^{2}$ eradication is still beyond reach, and the search for accessible and effective prevention and curing strategies along with significant investments is still a need to make it feasible.

In this context, nanoscience and nanotechnology are well placed to bring innovative solutions to the enormous challenge of winning the fight against DoP. ${ }^{3}$ Nanomaterials, which have been extensively focused on cancer-related biomedical applications for many years, are being intensively investigated to implement strategies to prevent and cure malaria, with the aim of overcoming the problems arising with conventional therapies. $^{4-6}$ Furthermore, as a vector borne parasitic disease, malaria can be faced at several stages of the infection process, from the mosquito vector to the infected erythrocytes, and these constitute different challenges to generate novel antimalarial strategies. Most efforts are addressed towards targeted drug-delivery systems, mostly including known antimalarial 
drugs. When developing anti-infective therapeutics, two essential objectives have to be fulfilled: delivering sufficiently high local doses that will rapidly eliminate the pathogen without allowing for it to evolve resistance, and doing so with overall doses sufficiently low so not to have side effects on the human host. Both goals can be simultaneously achieved through targeted drug delivery strategies that make use of nanocarriers (NCs). ${ }^{7,8}$ Many NCs have the capacity to encapsulate different types of drugs and of being functionalized with ligands targeting them to the desired cell type. In addition, if the NCs are loaded with large numbers of drug molecules to be delivered together, their antiparasitic effect will be potentiated and they will have a higher chance to quickly kill the infectious agent. NCs can be made having long shelf half-lives, low unspecific toxicity, large drug loading capacity, extended blood circulation times and safe elimination mechanisms.
In addition to therapeutic applications, nanomaterials could be ideally adapted to the development of new prevention strategies. Considering the difficulties in obtaining an efficient malaria vaccine, ${ }^{9}$ NCs are suited to be functionalized with several antigens, which might dramatically boost the prospects of obtaining a stronger immune response than with the current classical vaccination approaches.

Mosquito vector control constitutes an essential issue for malaria prevention since it can be highly effective to prevent transmission. The use of nets treated with insecticides and indoor spraying are the most frequent protection measures. However, the mosquito can be also reached through nanomaterials. The antimicrobial activity of metals such as silver, gold or platinum can be enhanced in biosynthesized nanostructured particles, which show up as a potential prevention weapon to hamper malaria transmission. ${ }^{10,11}$

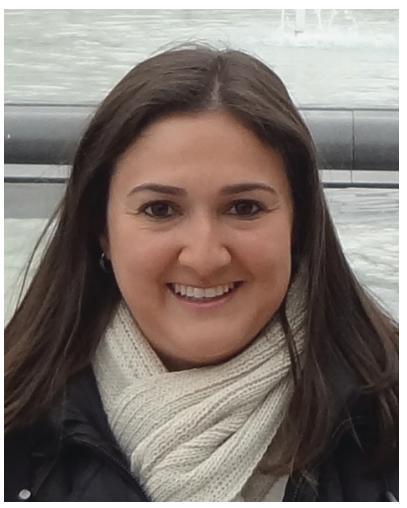

Livia Neves BorghetiCardoso
Livia Neves Borgheti-Cardoso graduated in PharmacyBiochemistry at the University of São Paulo (USP), Brazil. She got her Master (2012) and PhD (2016) degrees in Science at the USP with one-year as a PhD visitor at the University Medical Center Utrecht, The Netherlands, working on the development of organogels for the treatment of skin cancer by gene therapy. She received the Honorable Mention Award of the 2017 Capes Thesis Award, Brazil. In 2017 she joined the Nanomalaria Group as a Marie Skłodowska-Curie Actions fellow, with a research project based on the exploration of extracellular vesicles as a drug delivery system. She is currently a postdoctoral researcher at the Biomimetic Systems for Cell Engineering Group, IBEC.

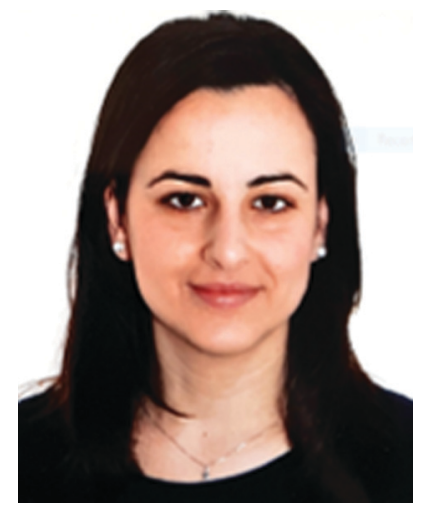

María San Anselmo got her degree in Biochemistry at University of Zaragoza in 2014. In 2015 she studied for a Master degree in Molecular and Cellular Biology and enjoyed a research grant in Taiwan. She joined the Liquid Crystals and Polymers group in 2016, where she is developing her PhD in Chemistry at INMA in the field of dendritic nanocarriers for biomedical applications.
María San Anselmo

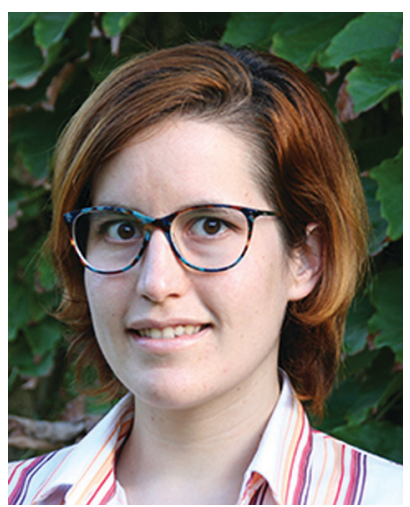

Elena Lantero
Elena Lantero is a Biotechnology graduate from the Universitat de València (2013), with a Master degree in Nanostructured Materials for Nanotechnology Applications obtained at the Universidad de Zaragoza (2015). She is currently finishing her PhD in the Nanomalaria Group focused on targeting strategies against Plasmodium, which has included two three-month research stays (2018 and 2019) at the Institute of Hygiene and Tropical Medicine (IHMT) in Lisbon.

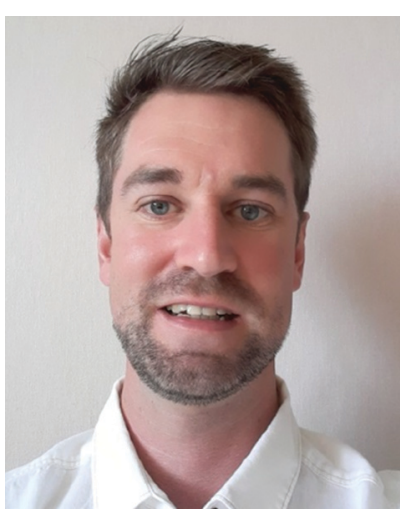

Alexandre Lancelot received his PhD in Chemistry in 2017 at the University of Zaragoza (Spain) where he studied dendritic materials for applications in nanomedicine. Nowadays, he is a postdoctoral researcher at the Autonomous University of Barcelona (Spain). His research interests focus on organic adhesives and coatings using green chemistry principles.
Alexandre Lancelot 
This review collects the research done on different types of nanomaterials which can be adapted to implement promising antimalarial tools with therapeutic or prophylactic roles. Before discussing the most relevant results in the literature, this review includes sections dedicated to the main characteristics of malaria infection and those challenges that nanomaterials face to constitute effective weapons against malaria.

\section{Malaria}

Malaria is a parasitic disease caused by Plasmodium spp. Among the more than 120 species in this genus, five of them (Plasmodium falciparum, Plasmodium vivax, Plasmodium malariae, Plasmodium

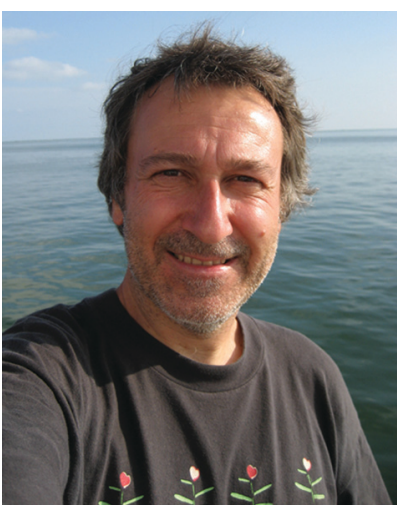

Xavier Fernàndez-Busquets
Xavier Fernàndez-Busquets graduated in Biochemistry at the Universitat Autònoma de Barcelona (1986), where he also obtained his $P h D$ in the field of Molecular Biology (1992). Between 1992 and 2001 he held several postdoctoral positions, among which are those at the Friedrich Miescher Institut (Novartis AG, Basel) and at the Woods Hole Marine Biological Laboratory (Massachusetts, US), where he worked on the evolutionary origins of specific cell adhesion and started his training in Nanotechnology. He obtained in 2001 a 5 year tenure track Ramón y Cajal position at the Universitat de Barcelona where he led a group focused on nanotechnological approaches to amyloid diseases and malaria. He became in 2006 Associate Researcher at the Institute for Bioengineering of Catalonia (IBEC) and since 2010 he is Head of the Nanomalaria Group (IBEC/ Barcelona Institute for Global Health, ISGlobal). www. fernandezbusquets.eu. ovale, and Plasmodium knowlesi) cause malaria in humans, $P$. falciparum and $P$. vivax being those involved in global malaria. ${ }^{12,13}$ P. falciparum is the species responsible for the most severe and potentially fatal form of malaria, whereas $P$. vivax has dormant liver stage parasites (hypnozoites) that can trigger a fullyfledged infection after months or even years remaining in a quiescent intrahepatocytic state. ${ }^{4}$

Plasmodium is an intracellular parasite with a complex life cycle shared between the human host and a mosquito vector from the Anopheles genus. When an infected mosquito bites a human, Plasmodium sporozoites (Fig. 1.1) that reside in the salivary glands are released into the blood stream and reach the liver, where they infect hepatocytes and multiply inside them (Fig. 1.2). Subsequently, parasite forms called merozoites are released into the blood, where in less than a minute ${ }^{14}$ they invade red blood cells (RBCs) and mature inside them. Ring stages grow into trophozoites as they feed on hemoglobin, and later divide their nuclei to form schizonts, from which the next generation of merozoites egresses to reinvade new RBCs, thus perpetuating the so-called intraerythrocytic cycle (Fig. 1.3). These stages of the parasite are responsible for all malaria symptoms, such as periodic fevers resulting from the immune response to the parasite,,$^{15}$ or more severe conditions such as placental malaria ${ }^{16}$ or capillary occlusion, ${ }^{17}$ which are derived from parasite sequestration in capillaries ${ }^{18}$ and from a phenomenon called rosetting, i.e. the adhesion between parasitized RBCs (pRBCs) and naïve RBCs to form cell aggregates termed rosettes.

A small number of pRBCs differentiate in each cycle towards the formation of gametocytes (Fig. 1.4), ${ }^{19}$ sexual forms of the parasite that sequester in the bone marrow until they mature and return to the circulation. When removed with a mosquito blood meal, gametocytes egress from the pRBC and become gametes. Fertilization produces a zygote (Fig. 1.5) that becomes a motile ookinete, which leaves the blood bolus to reach and cross the mosquito midgut endothelium, where it differentiates into an oocyst (Fig. 1.6) that grows to spawn sporozoites. These close the cycle by travelling through the haemolymph towards

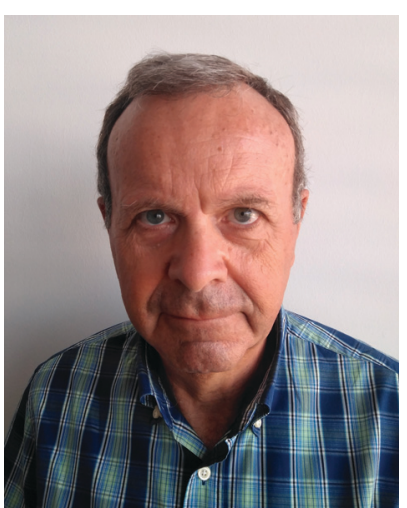

José Luis Serrano
José Luis Serrano has been a Professor of Organic Chemistry (University of Zaragoza) since 1996. He is director of the research group "Liquid Crystals and Polymers" (CLIP), which was recognized as Group of Excellence by the Government of Aragon. In 2017 he was awarded the highest prize of the Russian LC Society (Sodruzhestvo) "Frederiks medal", for the outstanding results in the field of Chemistry of Liquid Crystals. https://liquidcrystals. unizar.es/.

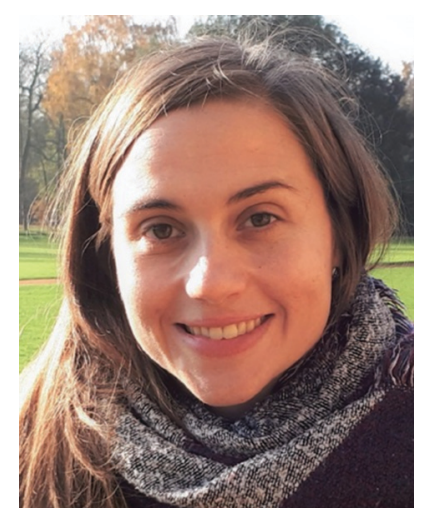

Silvia Hernández-Ainsa
Silvia Hernández-Ainsa completed her PhD in Chemistry in 2011 at the University of Zaragoza (Spain) in the area of dendritic materials. She then joined the University of Cambridge (UK) to work on nanopores for sensing and on DNA nanotechnology. Since 2017 she is ARAID researcher working at INMA (CSIC-University of Zaragoza). Her current research focuses on the development of DNA-based structures and other organic materials for applications in nanobiotechnology. 


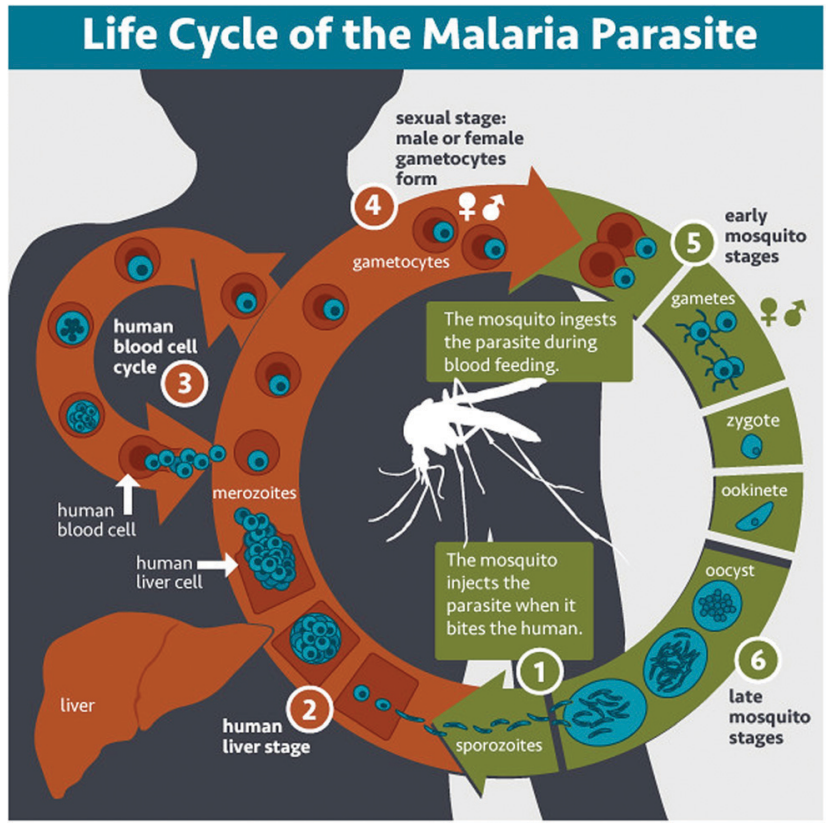

Fig. 1 Life cycle of Plasmodium. In a mosquito bite, sporozoites are injected (1), invade hepatocytes and divide inside them (2). Then merozoites are released into the blood and invade erythrocytes, growing and reinvading new RBCs in the intraerythrocytic cycle (3). Some merozoites differentiate into gametocytes (4), which are taken in another mosquito blood meal, and egress from the pRBC, forming the gametes that fuse into the zygote (5). The zygote develops into an ookinete that traverses the mosquito midgut to form an oocyst (6) that will grow until releasing the infective sporozoites. Image courtesy of the National Institute of Allergy and Infectious Diseases (https://www.niaid.nih.gov/diseases-conditions/ malaria-parasite).

the mosquito salivary glands, where they are ready for new inoculation into a human host (Fig. 1.1).

\section{Challenges for drug delivery}

\subsection{Drugs}

There is currently a large variety of antimalarial drugs (Fig. 2), but their application is often limited due to the development of

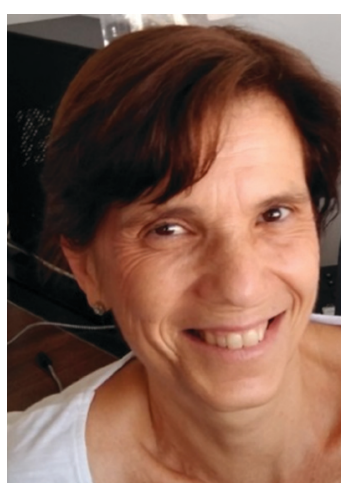

Teresa Sierra
Teresa Sierra received her PhD in Chemistry from the University of Zaragoza (Spain) and then she did a postdoctoral stay at the University of Colorado at Boulder, working on ferroelectric liquid crystals for NLO with Prof. D. M. Walba. Currently, she holds a position as Scientific Researcher at the INMA (CSIC-University of Zaragoza). Her research interests focus on exploiting liquid crystalline organization to attain functional materials and on dendritic materials for biomedical applications.

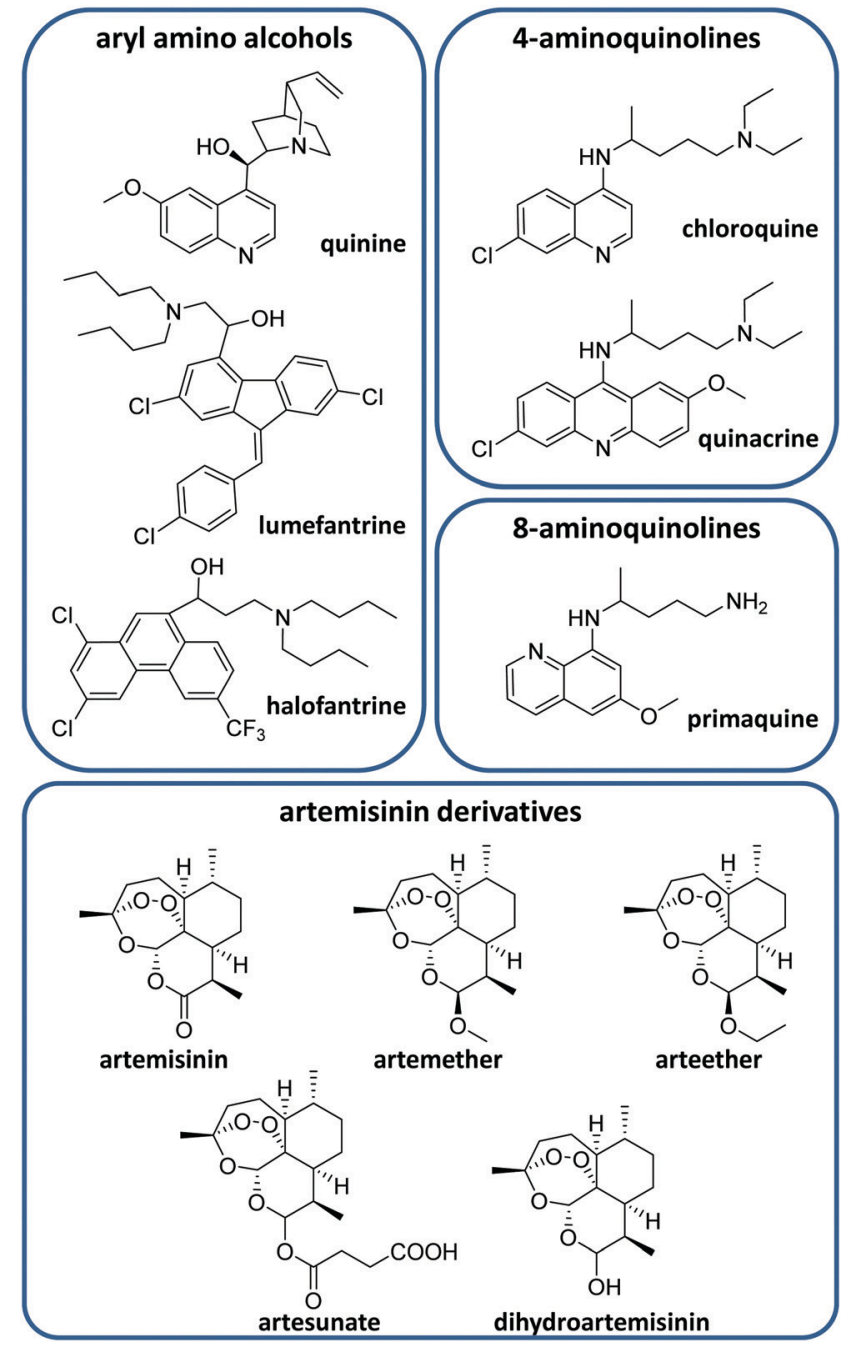

Fig. 2 Some antimalarial drugs discussed in this review.

resistance. In this regard, the WHO encourages the use of combination therapy, where two or more medicines with different mechanisms of action are used together, which helps to prevent or delay drug resistances. ${ }^{20}$ In this section, we present the most commonly used antimalarial drugs and briefly describe their distinct characteristics as well as precise antiplasmodial use.

Quinine (QN) was the first purified compound used to treat malaria and has been the most common and effective option to date. ${ }^{21}$ This drug is active against all Plasmodium spp. schizonts and the gametocytes of $P$. vivax, $P$. malariae and $P$. ovale but not against those of $P$. falciparum. ${ }^{20,22}$ Due to its reported resistance, QN is not currently used as the leading treatment but it is still employed for severe malaria. ${ }^{20,21} \mathrm{QN}$ is also recommended together with clindamycin in the treatment of uncomplicated malaria during the first trimester of pregnancy. ${ }^{20,23}$ This drug belongs to the aryl amino alcohol pharmacophore-type like lumefantrine, mefloquine, halofantrine or quinidine. Lumefantrine is another widely used drug, which is not administered alone but in combination with artemether (an artemisinin-derivative) to 
treat uncomplicated $P$. falciparum malaria in multiple doses. ${ }^{20}$ Due to its high lipophilicity, it requires being taken up within a fatty diet, which can represent a problem due to the difficulty in poor populations of having continuous access to such a meal. ${ }^{20}$ Mefloquine is commonly used as a chemoprophylactic for all types of malaria and in combination with artesunate (ATS) to treat uncomplicated malaria. $^{20}$

Other common active compounds against malaria include the 4-aminoquinolines (e.g. chloroquine, quinacrine, hydroxychloroquine, piperaquine, and amodiaquine) and the 8-aminoquinolines (primaquine, tafenoquine, and pamaquine). Chloroquine (CQ) is well absorbed from the gastrointestinal tract after oral administration. This drug is used to treat uncomplicated malaria caused by $P$. vivax, $P$. malariae and $P$. ovale in areas without CQ resistance. ${ }^{20}$ Primaquine (PQ) is the most remarkable drug belonging to the 8-aminoquinolines. It shows activity against $P$. vivax and $P$. ovale hypnozoites and it is generally administered together with artemisinin derivatives or $\mathrm{CQ}{ }^{20}$ Like other 8-aminoquinoline-type drugs, primaquine can entail risk for patients with glucose-6-phosphate dehydrogenase deficiency, which is unfortunately very common in malariaendemic areas. ${ }^{24}$

On the other hand, ATS, artemether, arteether and dihydroartemisinin are the most common artemisinin derivatives employed to treat malaria through artemisinin-based combination therapy (ACT). ${ }^{21}$ The most frequent ACT drug combinations to treat uncomplicated malaria are artemether/lumefantrine, ATS/ amodiaquine, dihydroartemisinin/piperaquine, ATS/mefloquine and ATS/sulfadoxine-pyrimethamine. ${ }^{25}$ Atovaquone/proguanil is a non-artemisinin-based combination therapy that is commonly employed for the prophylaxis of malaria and can be also used to treat CQ-resistant malaria. ${ }^{20,21}$

\subsection{Antimalarial drug distribution}

As the erythrocytic stage is responsible for all symptoms and pathologies of malaria, it is an obvious target for the administration of drugs. ${ }^{26}$ The bioavailability of antiplasmodial drugs is highly dependent on the administration route, the parenteral route being used for severe malaria, while oral delivery is the optimal strategy for uncomplicated malaria in order to maximize patient adherence to treatment. Some drugs like amodiaquine $^{27}$ and all artemisinin derivatives except ATS $^{28}$ have poor oral bioavailability, with below $35 \%$ of the ingested drug reaching the blood stream for most of them, thus requiring high dosages that could result in toxic side effects.

A second challenge for proper treatment is presented by the pharmacokinetic profile of antimalarial compounds, which has led to the implementation of combination therapies where, as explained above, two drugs are delivered together, one of them being usually an artemisinin derivative. In general, artemisinin derivatives have short half-lives $\left(t_{1 / 2}\right)^{29,30}$ and therefore the accompanying drug is usually long-lived $\left(t_{1 / 2}>4\right.$ days $)$ in order to achieve complete clearance of the parasite. This approach is, however, a matter of debate, because the time during which one of the drugs is present might be long enough to stimulate resistance evolution. ${ }^{31}$ Although CQ has an excellent absorption and circulation time profile, ${ }^{32}$ its widespread use in the past triggered the emergence of resistance. ${ }^{33}$ As a result, CQ is not widely used as first line treatment anymore, being only recommended in areas where resistances are not described in non-falciparum malaria. ${ }^{34}$

Antimalarials targeting the intraerythrocytic cycle have to face the challenge of RBC penetration. Mature erythrocytes do not endocyte, ${ }^{35}$ and drugs have to be passively absorbed to penetrate them. Although it is possible to efficiently load large amounts of drugs on RBCs ex vivo, it requires harsh manipulation such as hypotonic conditions. ${ }^{36}$ Drugs targeting RBCs should be hydrophobic to enhance their penetrability (the front-line drug artemisinin and its derivatives fulfill this requirement ${ }^{37}$ ), although hydrophobicity results in poor pharmacokinetics and off-target delivery, leading to accumulation in non-targeted tissues or interaction with plasma proteins. ${ }^{28,29}$

Loading drugs into RBCs and using these as vascular carriers is an attractive alternative because these cells have a large life span in the blood (of up to 120 days) and a large size, which prolongs the circulation time and permits the encapsulation of large amounts of drugs. Erythrocyte drug transporters limit extravasation, in addition to being biocompatible and safely removed from the body through natural mechanisms. ${ }^{38-40}$ As shown by Wilson et al., RBCs pretreated with the antimalarials halofantrine, lumefantrine, piperaquine, amodiaquine and mefloquine retained them inside, inhibiting the growth of the parasites that were invading drug-containing RBCs. ${ }^{38}$

\subsection{Drugs reaching Plasmodium}

Targeting Plasmodium in its liver phase represents an attractive prophylactic approach that would prevent the progress of the disease and its ensuing symptoms and disability caused by erythrocytic stages. Moreover, the administration of antimalarial drugs specifically to the liver could also be useful for $P$. vivax therapy. However, PQ is the only approved drug for hypnozoites, with the drawbacks that were discussed in Section 3.1.

Administration to Plasmodium early erythrocytic stages would be an interesting strategy to allow drugs a longer time to eliminate the parasite before it completes its intraerythrocytic development. However, delivering active compounds to RBCs infected with Plasmodium early stages is a challenging task, because the permeability of pRBCs to drugs remains low until $c a$. six hours after invasion. ${ }^{40,41}$

To reach their molecular targets inside pRBCs, antimalarial drugs must cross at least 3 membranes (the erythrocyte membrane, the parasitophorous vacuole and the parasite membrane), ${ }^{42}$ plus one extra lipid bilayer for those compounds having their target inside organelles such as the food vacuole or the apicoplast. Lipophilic drugs are able to cross these membranes through passive diffusion, whereas other compounds are carried inside the parasite by available channels or transporters. ${ }^{42}$ During Plasmodium intraerythrocytic infection and maturation, significant alterations in the structural and functional characteristics of RBCs are observed. ${ }^{43,44}$ Namely, Plasmodium changes the lipid and protein composition of the host cell plasma membrane in order to take up nutrients from 
the blood circulation, dispose of metabolic waste, and create and maintain electrochemical ion gradients. ${ }^{26,44}$ Around 10 to $20 \mathrm{~h}$ post-invasion, these alterations result in the formation of new permeation pathways, which allow the entry into parasitized RBCs of particles up to diameters of $70 \mathrm{~nm} \cdot{ }^{26,44}$ One transporter that is exported by the parasite to the pRBC membrane is the plasmodial surface anion channel, which contributes to nutrient acquisition for the intracellular parasites. ${ }^{45}$ Variation or differences in expression of this channel can be a source of potential resistances to the drugs relying on it. ${ }^{46-48}$

For those drugs able to cross the erythrocyte membrane, further penetration through the pasaritophorous vacuole or parasite plasma membranes seems to occur with more facility, as both have high capacity for permeating many solutes, ${ }^{42}$ possibly to facilitate intake of nutrients into the parasite. Penetration and a long residence time in certain target organelles are also important for several drugs to exert their antiparasitic action. In the case of $\mathrm{CQ}$, the main resistance mechanism consists of increasing drug efflux from the digestive vacuole, ${ }^{49}$ where CQ blocks the heme detoxification pathway. ${ }^{50}$ Mutations or gene duplications in transporter proteins can produce resistance to quinolines. ${ }^{49,51-53}$ Other biological features of Plasmodium can significantly reduce the drug amounts reaching target cells, thus favoring the evolution of resistance. In the murine malaria model Plasmodium berghei, spleen-sequestered pRBCs were less sensitive to artemisinin, ${ }^{54}$ likely because the limited diffusion of drugs into the organ resulted in final doses reaching the parasite that were much smaller than those found in the blood circulation.

\section{Nanomaterials against malaria}

As discussed in the following sections, nanomaterials and nanotechnology offer good opportunities for the development of customized nanocarriers capable of trapping antimalarial drugs as well as exerting targeted delivery. We include below several subsections in which antimalarial nanomaterials of different nature are discussed. Namely, nanocarriers built with lipids, polymers and dendrimers as well as inorganic NPs are presented. In the final subsection, we describe some other molecular platforms that do not belong to the previous categories but also display interesting potential for innovative antimalarial strategies.

\subsection{Liposomes}

Liposomes are artificial lipid bilayer vesicles containing an aqueous core formed by the self-assembly of phospholipids in aqueous media. ${ }^{4,55-57}$ Since their description in 1965, they have been extensively and successfully used as a drug delivery system. Due to their dual hydrophilic/hydrophobic nature, they can encapsulate water-soluble drugs in the aqueous core and lipophilic compounds in the phospholipid bilayer. ${ }^{4,57}$ Liposomes are versatile NCs with diverse properties depending on the size, phospholipid composition (Fig. 3), surface charge, and functionalization with diverse ligands. ${ }^{58,59}$ This versatility permits

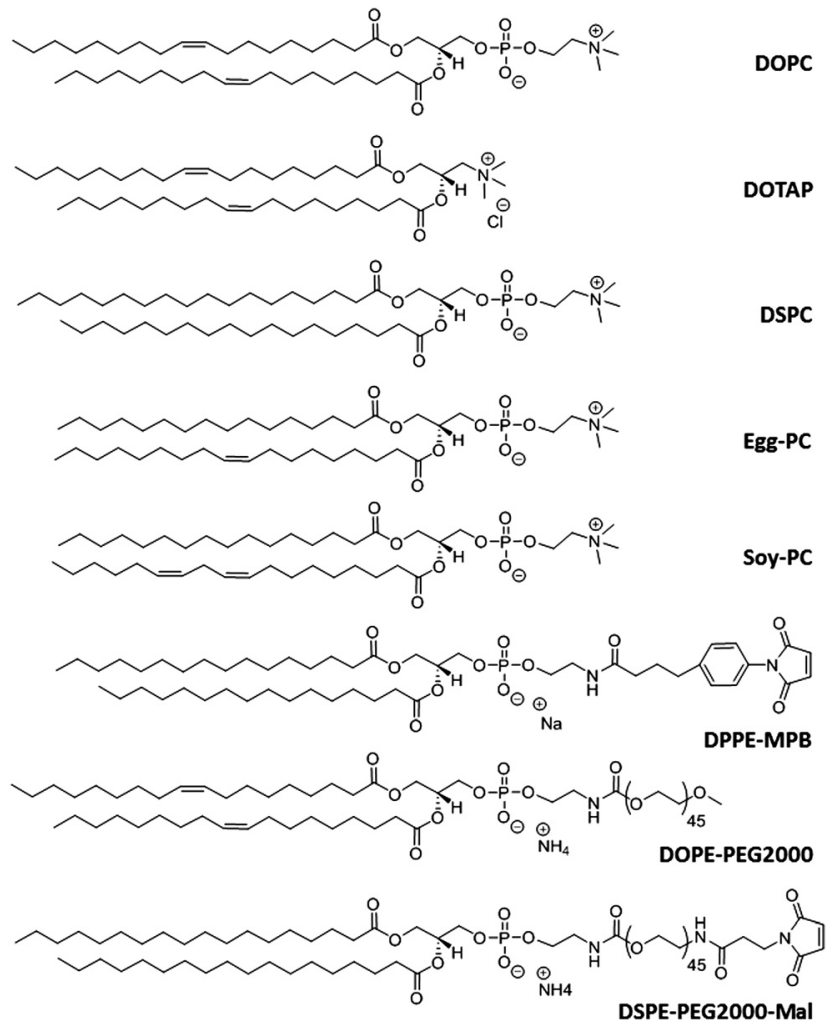

Fig. 3 Chemical structure of some phospholipids used in liposome formulation for malaria therapy. 1,2-Dioleoyl-sn-glycero-3-phosphocholine (DOPC), 1,2-dioleoyl-3-trimethylammonium-propane (DOTAP), 1,2-dis tearoyl-sn-glycero-3-phosphocholine (DSPC), L- $\alpha$-phosphatidylcholines (Egg-PC and Soy-PC), 1,2-dipalmitoyl-sn-glycero-3-phosphoethanolamine$\mathrm{N}$-[4-(p-maleimidophenyl)butyramide] (DPPE-MPB), 1,2-dioleoyl-sn-glycero3-phosphoethanolamine- $N$-[methoxy(polyethylene glycol)-2000] (DOPE-PEG 2000), and 1,2-distearoyl-sn-glycero-3-phosphoethanolamine- $N$-[maleimide (polyethylene glycol)-2000] (DSPE-PEG2000-Mal)

modulating their structure to protect drugs from chemical degradation, increase their solubility, prolong and sustain drug release, reduce toxicity and target cells specifically. ${ }^{60-62}$

Liposomes have been successfully administered by parenteral routes, but their oral administration is hampered by several obstacles such as instability in the gastrointestinal tract and difficulties in crossing biomembranes. By modifying the compositions of the lipid bilayers and adding polymers or certain ligands, both the stability and permeability of liposomes can be significantly improved for oral drug delivery. ${ }^{63}$ For instance, Coma-Cros et al. used the anionic copolymer Eudragit ${ }^{\circledR}$ S100 to assemble liposomes incorporating curcumin and containing either hyaluronan (Eudragit-hyaluronan liposomes) or the water-soluble dextrin Nutriose ${ }^{\circledR}$ FM06 (Eudragitnutriosomes). As a result, curcumin incorporated into Eudragitnutriosomes enhanced its antimalarial activity upon oral administration to Plasmodium yoelii-infected mice. Eudragitnutriosomes presented better performance than Eudragithyaluronan liposomes due to the greater stability of the former, as shown by the vesicle behavior in gastrointestinal fluids. ${ }^{64}$

Several studies have tried to target liposomes encapsulating PQ to the liver stage of malaria. ${ }^{65}$ In $P$. berghei infections in 
mice, liposomes could decrease the toxicity of parenteral PQ delivery, which allowed for the administration of higher doses and resulted in the cure of the animals following a single intravenous injection. ${ }^{66}$ Tissue distribution studies demonstrated that PQ encapsulated in liposomes accumulated mainly in the liver and spleen and not so much in the kidneys, lungs, heart, or brain, being eliminated more slowly than its free counterparts. ${ }^{67,68}$ Although peptide- and amino acidconjugated derivatives of $\mathrm{PQ}$ encapsulated in liposomes did not increase the therapeutic effect in comparison to free $\mathrm{PQ}^{66}$ they presented a reduction of toxicity. ${ }^{68}$ In another study, liposomes loaded with antimalarial drugs (especially PQ) were engineered to contain a galactosyl glycolipid that specifically targeted a galactose-binding lectin in hepatocytes. These experiments showed a remarkable phenomenon: drug-free liposomes had a curative effect on sporozoite-induced murine malaria, likely due to the competition of liposomes and sporozoites for the same receptor. ${ }^{69}$ In addition, considering the reduced doses of $\mathrm{PQ}$ required to achieve a curative effect, it was estimated that the efficacy of PQ was enhanced more than 46000 times by its encapsulation into liposomes containing the galactosyl glycolipid. ${ }^{70}$

To specifically target the liver in vivo, Longmuir et al. formulated a liposome bearing a 19-amino acid sequence present at the surface of the $P$. berghei circumsporozoite protein. This liposome successfully reached the liver following intravenous administration in mice. ${ }^{71}$ Despite these promising results, liver-targeted-liposomes have not progressed towards a strategy to fight malaria, probably because of the lack of sufficiently specific markers for Plasmodium-infected hepatocytes. ${ }^{43}$ As a consequence, most current studies using liposomes to deliver antimalarial drugs target the erythrocytic stage.

Essentially, liposomes can be developed to deliver drugs to the pathogen's erythrocytic forms at three different times: (i) before parasite infection, (ii) to RBCs infected with Plasmodium early stages, and (iii) to RBCs infected with Plasmodium late stages. The administration of a drug for each of these phases has its own advantages and challenges. Regardless of the time when liposomes will deliver the drug, the lack of endocytic processes in RBCs is one of the main obstacles to be dealt with. In a proof of concept study, quantum dots encapsulated in liposomes allowed the visualization of a homogeneous distribution of liposome content within P. falciparum-infected RBCs. This indicated that the liposomal cargo entered the cell through fusion of the liposome bilayer with the cell plasma membrane. ${ }^{72}$ It has been reported that the fusion of liposomes with mammalian cells is significantly dependent on the lipid composition and fluidity of the liposome membrane. ${ }^{44}$ For instance, the efficacy of stearylamine in inhibiting the growth of $P$. falciparum was reduced when incorporated in sphingomyelin liposomes compared to either egg phosphatidylcholine (Egg-PC) or soy phosphatidylcholine (Soy-PC) liposomes. This probably occurred due to reduced fusion/ interaction of pRBCs with rigid sphingomyelin-containing liposomes compared to fluid Egg-PC or Soy-PC liposomes. ${ }^{44}$ Thus, to favor fusion events, it is advisable to formulate liposomes with relatively fluid lipid bilayers, although this incurs the risk of leaking for small drugs encapsulated in the liposomal aqueous core. On the other hand, liposomes formulated with saturated lipids retain drugs better because they have less fluid bilayers, but at the cost of decreased membrane merging with target cells. ${ }^{39}$ In both cases, the amount of delivered drug will get reduced, and a balance must be found to limit drug leakage while allowing lipid bilayer fusion events.

Antibodies have been successfully used to target pRBCs. ${ }^{43,73-76}$ Antibody-targeted liposomes containing on their surface $\mathrm{F}\left(\mathrm{ab}^{\prime}\right)_{2}$ fragments of a mouse monoclonal antibody raised against $P$. berghei-infected mouse erythrocytes significantly increased the therapeutic efficacy of CQ, showing that target-specific liposomes can cure CQ-resistant malarial infections. ${ }^{73}$ Urbán et al. also observed an improvement of up to tenfold in the therapeutic effect of CQ when delivered inside liposomes covalently functionalized with oriented, specific half-antibodies against $P$. falciparum late form-infected RBCs. ${ }^{43}$ Further studies showed that this same targeted liposome similarly improved the in vitro efficacy of fosmidomycin, another antimalarial drug. ${ }^{74}$ Immunoliposomes formulated using as a targeting molecule a polyclonal antibody against the NTS-DBL1 $\alpha$ N-terminal domain of $P$. falciparum erythrocyte membrane protein 1 (PfEMP1) and encapsulating lumefantrine were also used to specifically recognize and disrupt rosettes while simultaneously eliminating those pRBCs forming them. ${ }^{76}$ As shown by these studies, antibodyfunctionalized liposomes can discriminate pRBCs from noninfected RBCs and specifically deliver antimalarial drugs to pRBCs. However, some issues limit the use of antibodies as targeting molecules in antimalarial therapy, such as the elevated cost of production, ${ }^{77}$ the high immunogenicity unless genetically engineered humanized forms are used, and the potential decrease in targeting efficiency due to the variability of Plasmodium proteins exposed on pRBC surfaces. ${ }^{26}$

Past research has paid little attention to the development of liposomes to deliver a drug to RBCs before Plasmodium infection; however, this strategy could represent an interesting prophylactic approach that deserves further study. Moles et al. developed an immunoliposome bearing antibodies against the RBC surface protein glycophorin A that targeted all RBCs, both naïve and Plasmodium-infected. ${ }^{39}$ In addition to targeting the parasite, this approach permitted loading of drugs into RBCs prior to infection. To improve the targeting properties, a lipid bearing a $2 \mathrm{kDa}$ polyethylene glycol (PEG) linker terminated with a maleimide group (Mal) was included in the liposome formulation aiming to facilitate antigen access to targeting antibodies (Ab) and to enhance the coupling reaction sterically hindered in the original immunoliposome by the polar heads of surrounding lipids. Three types of liposomes (LP) were designed and the linker was used for the oriented binding of half-antibodies through their free thiols (LP-PEGMal-Ab) or of whole antibodies through their carbohydrate (CHO) moieties present in antibody Fc regions (LP-PEG-Mal$\mathrm{CHO}-\mathrm{Ab}$ ), and for non-oriented binding through primary amino groups on $\mathrm{Ab}$ amino acid residues (LP-PEG-Mal- $\mathrm{NH}_{2}-\mathrm{Ab}$ ). The type of binding influenced the cell targeting efficiency 
(LP-PEG-Mal-NH ${ }_{2}$-Ab $\gg$ LP-PEG-Mal-CHO-Ab $\gg$ LP-PEG-Mal-Ab). ${ }^{39}$ These glycophorin A-targeted liposomes loaded with CQ were highly efficient in transferring the drug into RBCs, as demonstrated by a complete inhibition of in vitro parasite growth after only 15 min of incubation with the immunoliposomized drug, whereas after the same time an equal amount of free CQ had no observable effect on the parasite viability. In this scenario, the parasite encountered the drug immediately after entering the host cell, which drastically compromised its survival capacity. ${ }^{39}$

Although the administration of active compounds to RBCs is a promising prophylactic strategy, some requirements have to be considered, including (i) determination of a maximum immunoliposome concentration in vivo to avoid RBC agglutination, ${ }^{39}$ (ii) restriction to drugs that do not interfere with RBC physiology, ${ }^{78}$ and (iii) use of liposome formulations that will merge with the RBC before the cell is removed by the spleen due to alterations induced by liposomes adsorbed on its surface. $^{39}$

Since only after around $24 \mathrm{~h}$ post-invasion will Plasmodium export a substantial number of receptors and transporters to the host cell plasma membrane, the design of liposomes specifically targeted to early blood stages has remained remarkably elusive. ${ }^{40}$ Most published studies report the development of liposomes for delivering an active compound to late parasite stages, ${ }^{26,39,43,60,73-76,79-84}$ when significant alterations in the structural and functional characteristics of RBCs are observed. ${ }^{43,44}$ These liposomes have been formulated to deliver a high amount of drug to the targeted site to compensate for the short time available to kill the parasite before its egress. Different strategies can be used alone or in combination to guarantee an optimal therapeutic drug dose (as depicted in Fig. 4), including approaches to improve the encapsulation efficiency, ${ }^{39,75,83}$ to efficiently target RBCs infected with Plasmodium late stages ${ }^{26,43,73-76,84}$ and to increase the circulation time of liposomes. ${ }^{80-82}$ In addition, combination therapies contemplating the simultaneous administration of two or more active compounds have also been proposed. ${ }^{60,79}$ The encapsulation efficiency in liposomes of many antimalarial drugs can be increased using active loading techniques based on $\mathrm{pH}$ gradients. ${ }^{39,75,83}$ Drugs with amphiphilic nature such as CQ and $\mathrm{PQ}$, which depending on the solution $\mathrm{pH}$ present different degrees of protonation, can be efficiently encapsulated using a pH gradient. ${ }^{75,83}$ The majority of antimalarial drugs under development are lipophilic with poor plasma solubility and large biodistribution volumes and, consequently, with low accumulation in RBCs. ${ }^{57,75}$ Active encapsulation in liposomes using a citrate-buffered $\mathrm{pH}$ gradient allowed for the stable incorporation of hydrophobic drugs containing weakly basic ionizable moieties, which accumulate in the inner liposome membrane leaflet. Aryl amino alcohol- and 4-aminoquinoline-type drugs were efficiently delivered to $P$. falciparum-infected RBCs upon encapsulation into liposomes targeting all RBCs, inhibiting parasite growth in vitro and exhibiting considerable improvements in drug activity when compared to the free administered drug after only $15 \mathrm{~min}$ of exposure to $P$. falciparum cultures. $^{75}$

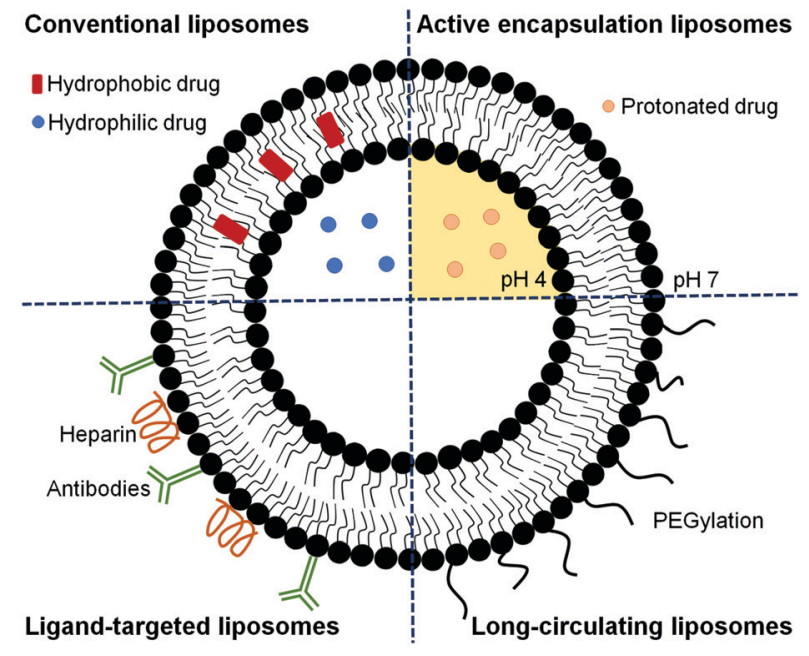

Fig. 4 Schematic representation of liposomes formulated using different strategies aiming at improving the therapeutic efficacy of encapsulated drugs. Conventional liposomes are formed by phospholipids and cholesterol and can encapsulate hydrophilic drugs in their aqueous core and hydrophobic compounds in their phospholipid bilayer. Active encapsulating liposomes hold a $\mathrm{pH}$ gradient that can improve the loading of drugs with amphiphilic nature, which, depending on the $\mathrm{pH}$, are found in their protonated or deprotonated forms. Ligand-targeted liposomes are developed using specific pRBC ligands such as antibodies or heparin and promote the delivery of high drug doses with few side effects. Long-circulating liposomes can be formulated by modifying the surface of liposomes with PEG to enhance the blood residence time.

As interesting alternatives to antibody-mediated targeting, certain glycosaminoglycans like heparin, heparan sulfate and chondroitin sulfate ${ }^{84}$ are being recognized as one of the main pRBC-binding molecules. ${ }^{26}$ Heparin bound to liposomes has dual activity as a pRBC targeting molecule acting mainly on trophozoites of some PfEMP1-expressing lines, ${ }^{85}$ and on schizont stages, ${ }^{86}$ and as an antimalarial drug it blocks merozoite invasion of erythrocytes. ${ }^{87}$ In addition, heparin is significantly less expensive to obtain than monoclonal antibodies, resulting in heparin-liposomes having a cost about ten times lower than immunoliposomes of similar targeting activity. ${ }^{84}$ In addition, and most importantly, no resistance to heparin as an antimalarial drug has been described so far. ${ }^{87}$ Furthermore, it has been shown that the anticoagulant activity of heparin when covalently bound to liposomes was substantially reduced. ${ }^{84}$ Heparin used at non-anticoagulating concentrations electrostatically adsorbed onto positively charged liposomes enhanced threefold the activity of encapsulated $\mathrm{PQ}$ in in vitro $P$. falciparum cultures.

Heparin-related polysaccharides such as heparan sulfate ${ }^{88,89}$ and chondroitin sulfate ${ }^{90}$ could also be used as targeting molecules and, compared with heparin, they have the advantage of having a much lower anticoagulant activity. ${ }^{84}$ Marques et al. used single-molecule force spectroscopy to measure the binding forces between chondroitin 4-sulfate and pRBCs or non-infected RBCs deposited on poly-L-lysine-coated glass slides. ${ }^{84}$ Although specificity of adhesion of these polysaccharides to pRBCs has been observed, challenges related to the 
attachment of a ligand on the liposome surface in a reproducible manner under good manufacturing practice conditions should be considered and stability requirements have to be optimized. ${ }^{91}$

Long-circulating liposomes can considerably enhance the blood residence time, improve the release profile of the drug at the site of infection, and facilitate a better interaction with infected RBCs, resulting in the inhibition of parasite growth to a higher extent. ${ }^{4,80}$ It has been shown that the interaction of PEG-grafted liposomes with pRBCs depends on the density of the polymer and the optimal chain length. ${ }^{80}$ Rajendram et al. demonstrated that the circulation life of the drug monensin can be modulated using PEG-functionalized lipids and liposomes modified with different densities of distearoyl phosphatidylethanolaminemethoxy-PEG 2000 (DSPE-mPEG2000), which enhanced the antimalarial efficacy of liposomal formulations compared with that of free monensin. This study also reported that the coadministration of stearylamine, monensin, and artemisinin with 5\% DSPE-mPEG-2000 could completely eliminate the parasite burden. ${ }^{80}$ It has also been shown that the antiplasmodial activity of stearylamine in liposomes has the maximum effect when delivered through Soy-PC liposomes. The inhibition of parasite growth was regulated by the chain length of the alkyl group and the density of stearylamine in liposomes. In addition, the incorporation of either cholesterol or DSPE-mPEG-2000 in Soy-PC-stearylamine liposomes improved their efficacy. ${ }^{44}$ Liposomes were also used to improve the short half-life and poor bioavailability of $\operatorname{artemisinin}^{81}$ and its derivatives, such as ATS. ${ }^{82}$ Artemisinin loaded in conventional and PEG-containing liposomes showed a much longer blood circulation time than free artemisinin, being detectable 3 and $24 \mathrm{~h}$ after administration, respectively, while free artemisinin was rapidly cleared from the plasma and barely detected $1 \mathrm{~h}$ after administration. ${ }^{81}$ Hydrophobic ATS was conjugated with hydrophilic glycerophosphorylcholine as a linker to synthesize an ATS-based dimeric conjugate. The conjugate was assembled into liposomes with a high drug loading of $77.6 \%$, exhibiting a longer blood half-life and superior in vitro antimalarial activity compared to free ATS and conventional ATS-loaded liposomes, without causing hemolysis. ${ }^{82}$

The simultaneous administration of several drugs usually improves their separate antimalarial effects, ${ }^{60,92}$ and liposomes, due to their structure, are ideally suited for such combination therapies at the nanoscale. ${ }^{77,84}$ Curcuminoids, which are poorly water-soluble compounds with promising antimalarial activity, were loaded into liposomes for intravenous administration and combined with the intramuscular administration of $\alpha / \beta$ artemether, which is known to have a short half-life in the body. This combination therapy was able not only to cure $P$. berghei-infected mice but also prevented recrudescence. ${ }^{60}$ Nevertheless, combined drugs exhibit different pharmacokinetic profiles and are often eliminated from the circulation at different rates, which decreases the treatment efficacy. However, if drugs are delivered encapsulated together, they will be removed from the organism simultaneously with their carrier, thus sustaining constant relative ratios throughout the treatment. Based on this concept, a glycophorin A-targeted immuno-PEG-liposome was formulated to encapsulate the hydrophilic antimalarial pyronaridine in its aqueous core and the lipophilic compounds domiphen bromide or atovaquone in its lipid bilayer. When tested in in vitro $P$. falciparum cultures, this strategy offered antiparasitic activities significantly higher than when the drugs were administered together in their free forms. ${ }^{79}$

Despite the considerable therapeutic advantages of liposomes, their clinical translation to antimalarial treatments has not progressed as quickly as the encouraging positive preliminary results predicted. The main issues behind this delay are pharmaceutical manufacturing limitations, government regulations and intellectual property. ${ }^{93}$

\subsection{Polymers}

This section includes relevant studies reported in recent years focused on polymeric nanostructures developed for drug delivery systems, polymer-based prodrugs and also prophylactic tools. ${ }^{94}$ The last sub-section is devoted to dendrimers as a unique family of hyperbranched polymers with special features for drug conjugation.

4.2.1 Polymeric NCs for encapsulation and covalent conjugation of drugs. A group of cationic (AGMA1 and ISA1) and anionic (ISA23) polyamidoamines containing tert-amine, carboxyl, guanidine and/or hydroxyl groups (Fig. 5) have been studied by Fernàndez-Busquets et al. ${ }^{95}$ for the encapsulation and systemic delivery of CQ and PQ. In addition, these structures together with a cationic poly(amidoamino acid) containing L-arginine units, ARGO7, were thoroughly studied by the same group for the oral administration of CQ. ${ }^{96}$ Further traceability studies of the empty polyamidoamines labeled with fluorescein and orally administered to female Anopheles
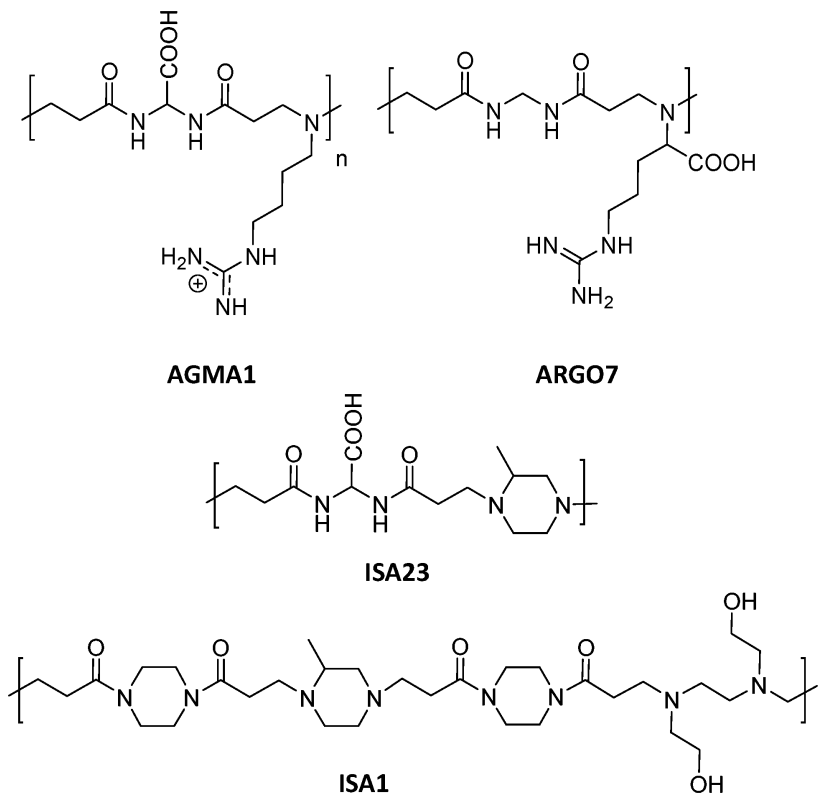

Fig. 5 Chemical structures of AGMA1, ISA23, ISA1 and ARGO7. ${ }^{95,96}$ 
gambiae mosquitoes postulated these polymeric platforms as good NCs to directly target Plasmodium in the mosquito vector. $^{97}$

Biodegradable synthetic polymers such as poly(D,L-lactic-coglycolic acid) (PLGA), polyalkylcyanoacrylates or poly(D,L-lactic acid) have been broadly used to prepare polymeric NPs. ${ }^{6,98}$ Some studies with PLGA NPs included the synergistic co-delivery of CQ and the antibiotic azithromycin ${ }^{99}$ (the most potent antimalarial macrolide widely studied in combination with other antimalarial drugs to improve their efficacy ${ }^{100}$ ) and the co-encapsulation of ATS and curcumin ${ }^{101}$ as promising combination therapies for malaria.

Another possibility explored is the use of nanocapsules composed of an oil core surrounded by a polymeric membrane. One example is found in the work of Haas et al., where poly( $(\varepsilon-$ caprolactone) (PCL) and polysorbate 80 (also called Tween 80) nanocapsules were used to deliver QN. ${ }^{102}$ Similarly, the antimalarial halofantrine was encapsulated into PCL nanocapsules, which resulted in an improvement of the cardiovascular parameters frequently altered by this drug. ${ }^{103}$ In this line, Velasques and co-workers developed polysorbate 80-coated PCL nanocapsules to co-encapsulate $\mathrm{QN}$ and curcumin, increasing the efficacy against $P$. falciparum at the same time that the cytotoxicity was reduced. ${ }^{104}$

A series of three hydroxypropylmethylcellulose (HPMC) polymers with different grades of acetyl (A) and succinyl (S) functionalization (HPMC-AS, Fig. 6), and the amphiphilic block copolymer PCL-block-poly(ethylene glycol) (PCL-b-PEG) were tested to improve the oral bioavailability of the synthetic antimalarial artefenomel mesylate (or OZ439). Specifically, the drug was coupled with an anionic species to form a hydrophobic ion pair complex, ${ }^{105}$ in an encapsulation process that was done through a scalable and cheap method called flash nano precipitation. Polymers with higher succinate contents displayed larger negative charge, whereas the introduction of acetyl moieties resulted in more hydrophobicity. Polymers with larger percentages of either succinate or acetyl did not show enhanced encapsulation efficiency, suggesting that both hydrophobic and ionic interactions (succinate groups on HPMC-AS) were required for stability. This was also demonstrated by the lack of stability provided by the non-charged amphiphilic

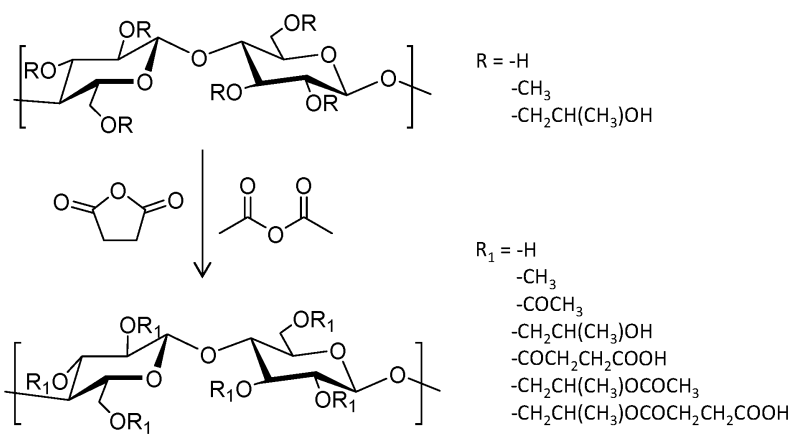

Fig. 6 General reaction scheme to convert HPMC polymers to HPMC-AS through the addition of acetyl and succinyl groups. ${ }^{105}$
PCL- $b$-PEG. Release experiments were done mimicking oral administration and in vivo conditions. These polymer-based NPs exhibited a sustained OZ439 release profile and significantly higher drug bioavailability compared to that of the unencapsulated drug.

Antisense oligodeoxynucleotides (ODN) represent an alternative to traditional antimalarial drugs. However, their low stability in plasma can hamper their therapeutic function. The potential of the positively charged chitosan polymer to stabilize ODN through the formation of polyplexes was explored by Foger et al., enabling ODN to show optimal interference with the expression of Plasmodium topoisomerase II, an enzyme necessary for parasite division after invasion of human erythrocytes. ${ }^{106}$ Further studies employed this natural aminoglucopyran to covalently conjugate artemisinin (Fig. 7), obtaining more soluble derivatives. ${ }^{107}$ This original strategy consisting of the covalent bonding of the drug to a polymer carrier was proposed in 1975 by Helmut Ringsdorf. ${ }^{108}$ Generally, an enzyme or pH-sensitive linker is employed to enable drug release once in the intracellular environment. ${ }^{24}$

Despite the scarce exploration of this strategy in the field of infectious diseases caused by a parasite, its successful use for leishmaniasis treatment ${ }^{109,110}$ encouraged its application to malaria since both pathologies share many features. ${ }^{24}$ PQ represents the most widely studied antimalarial drug within the polymer-drug conjugation approach. Already in 1986, PQ modified with a pendant cysteine group was linked through a disulfide bond to a protein carrier containing (pyridyldithio)propionate groups. ${ }^{111}$ More recently, PQ was also conjugated to poly- $\gamma$-glutamic acid (PGA) modified with a synthetic trivalent glyco-ligand (TriGalNAc) through an amide linkage, obtaining a TriGalNAc-PGA-PQ conjugate. ${ }^{112}$

This covalent approach has been also used for the conjugation of more than one drug onto the same polymer, a desirable
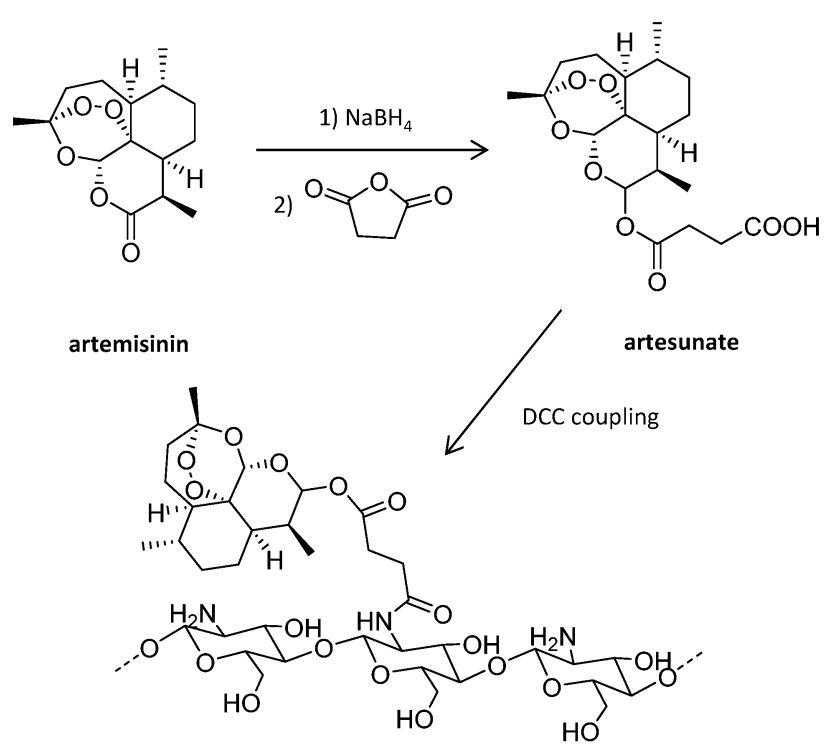

chitosan-artemisinin conjugate

Fig. 7 Synthesis of chitosan-artemisinin derivatives. ${ }^{107}$ 
strategy for combination therapy. An example is found in Kumar and co-workers' poly(organophosphazenes), an inorganic polymer designed as a nanocarrier for the co-delivery of $\mathrm{PQ}$ and dihydroartemisinin. ${ }^{113,114}$ Firstly, the polymeric backbone of poly(dichlorophosphazene) was functionalized with various substituents like isopropyl alcohol, 4-hydroxyacetanilide, 4-hydroxybenzaldehyde and ethyl 4-aminobenzoate, providing a wide variety of physicochemical properties. Then, the remaining unsubstituted polymer backbone was substituted stepwise with the two drugs to provide the polymer-linked combined antimalarial drug conjugates. All the prepared NPs showed negative surface charge (between -21 and $-32 \mathrm{mV}$ ) and a diameter between 128 and $179 \mathrm{~nm}$, and their antimalarial activity was improved compared to the standard drug combination.

Polymer NCs have been also investigated to improve the action of the antimalarial drug atovaquone, creating long-acting injectable formulations. Bakshi et al. prepared a library of solid drug NPs containing atovaquone and a binary combination of surfactant and polymer, including e.g. poly(ethylene oxide)80block-poly(propylene oxide)27-block-poly(ethylene oxide)80 (Pluronic F68), poly(vinylpyrrolidone) (PVP K30), HPMC, polyvinyl alcohol (PVA), and PEG. ${ }^{115}$ The most promising chemoprophylactic activity was shown for two nanoformulations containing the nonionic hydrophilic polymers PVP K30 and PVA.

4.2.2 Polymer-based RBC membrane nanomimics. An ingenious strategy developed by Najer et al. ${ }^{116}$ consisted in blocking the life cycle of the parasite by preventing the invasion of host RBCs. For that purpose, nanostructures mimicking RBC membranes, i.e. nanomimics, based on block copolymers with heparin as a host cell receptor were built (Fig. 8a).

The design of nanomimics involved the initial synthesis of two different block copolymers: poly(2-methyl-2-oxazoline)b l o $c k$-poly(dimethylsiloxane)-block-poly(2-methyl-2-oxazoline) (PMOXA- $b$-PDMS- $b$-PMOXA), which forms a polymersome, and the PDMS-heparin block copolymer, with the chain of the receptor heparin as a hydrophilic domain (Fig. 8b). Then, the nanomimic 3D structure (Fig. 8c) was obtained by bulk rehydration of a mixture of PMOXA- $b$-PDMS- $b$-PMOXA with $25 \%$ (w/w) PDMS-heparin. The resulting self-assembled structure exposed most of the heparin part outwards, making it accessible to bind parasite ligands involved in the initial attachment to host cells. These nanomimics have displayed a therapeutic effect without the need for any other substance by blocking invasion of host RBCs by the P. falciparum merozoite. Furthermore, they can also boost immune responses due to the increased number of free merozoites circulating, thus acting as vaccine adjuvants.

In a further study, ${ }^{117}$ the antimalarial activity of different nanomimics constructed by varying the number or length of heparin molecules on their surface was evaluated. Specifically, their interaction with a well-known heparin-binding protein on the surface of merozoites, $P$. falciparum merozoite surface protein 1, was measured, showing high binding affinity. These findings involving malaria parasite receptor molecules are very relevant for the optimization of future nanomimic therapies for infectious diseases.

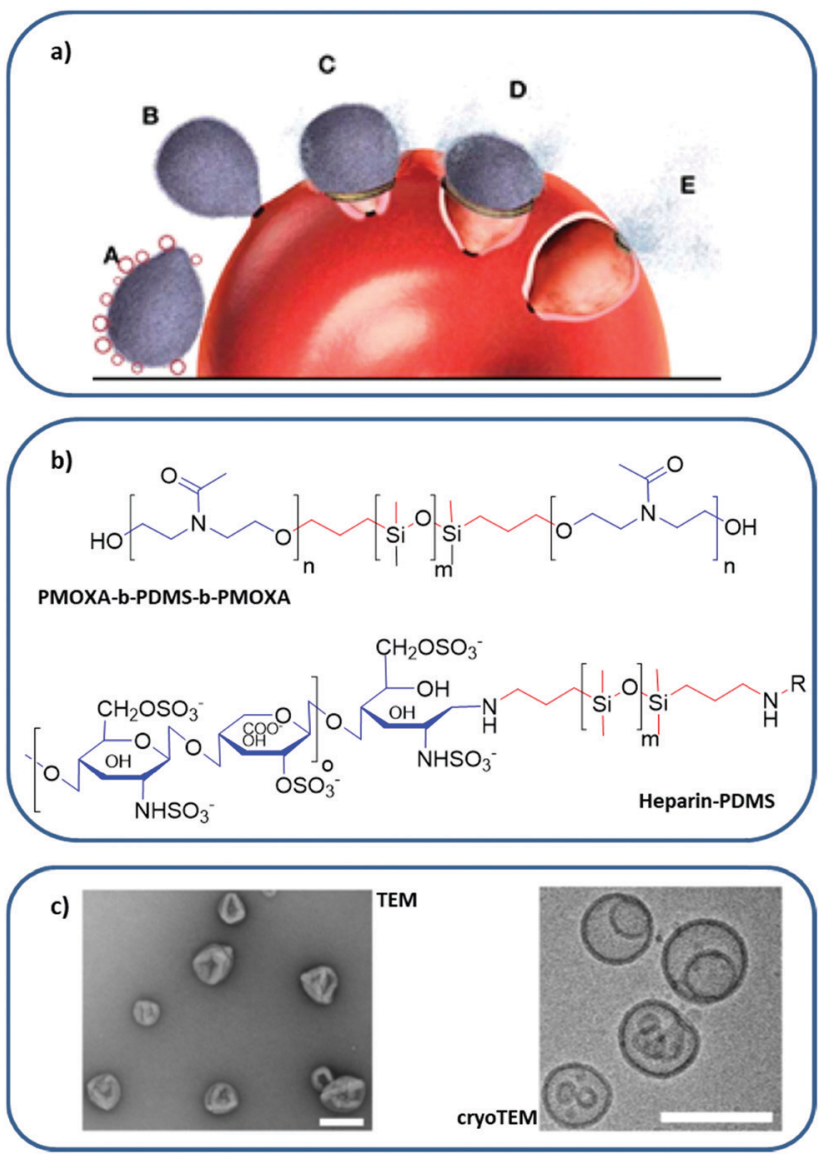

Fig. 8 Polymer-based RBC membrane nanomimic concept and characteristics: (a) schematic representation of the nanomimic concept. In step A, fresh nanomimic structures (red circles) block merozoites (gray oval structures) before they enter new RBCs (steps B, C, D, and E). (b) Chemical structure of PMOXA- $b$-PDMS- $b$-PMOXA and PDMS-heparin, where hydrophilic domains are colored in blue and hydrophobic cores in red. (c) TEM and cryo-TEM images of the nanomimics; scale bars: $200 \mathrm{~nm}$. ( $a$ and $c$ ) reprinted (adapted) with permission from Najer et al. ${ }^{116}$ copyright (2014) American Chemical Society.

4.2.3 Polymeric hydrogels. Polymeric hydrogels can be defined as water-swollen polymeric materials that maintain a distinct three-dimensional structure, which constitute the first biomaterials designed for use in the human body. ${ }^{118}$ Several methods for the preparation of polymeric hydrogels have been explored and they can exhibit different morphologies including slabs, microparticles, films and NPs. ${ }^{98}$ Various parameters determine the degree of porosity of polymeric hydrogels, such as the starting material or preparation method, which can influence their bioactivity.

An example of using hydrogels as antimalarial nanocarriers is the gum acacia-based hydrogels as a controlled dual-drug delivery system for 4-aminoquinolines and curcumin. ${ }^{119}$ The drug release profile of this system showed dependence on the degree of crosslinking of the hydrogel network and exhibited different pharmacokinetics for both drugs, which may contribute to overcoming undesirable drug resistance. Another study developed by Aderibigbe ${ }^{120}$ comprised soy 


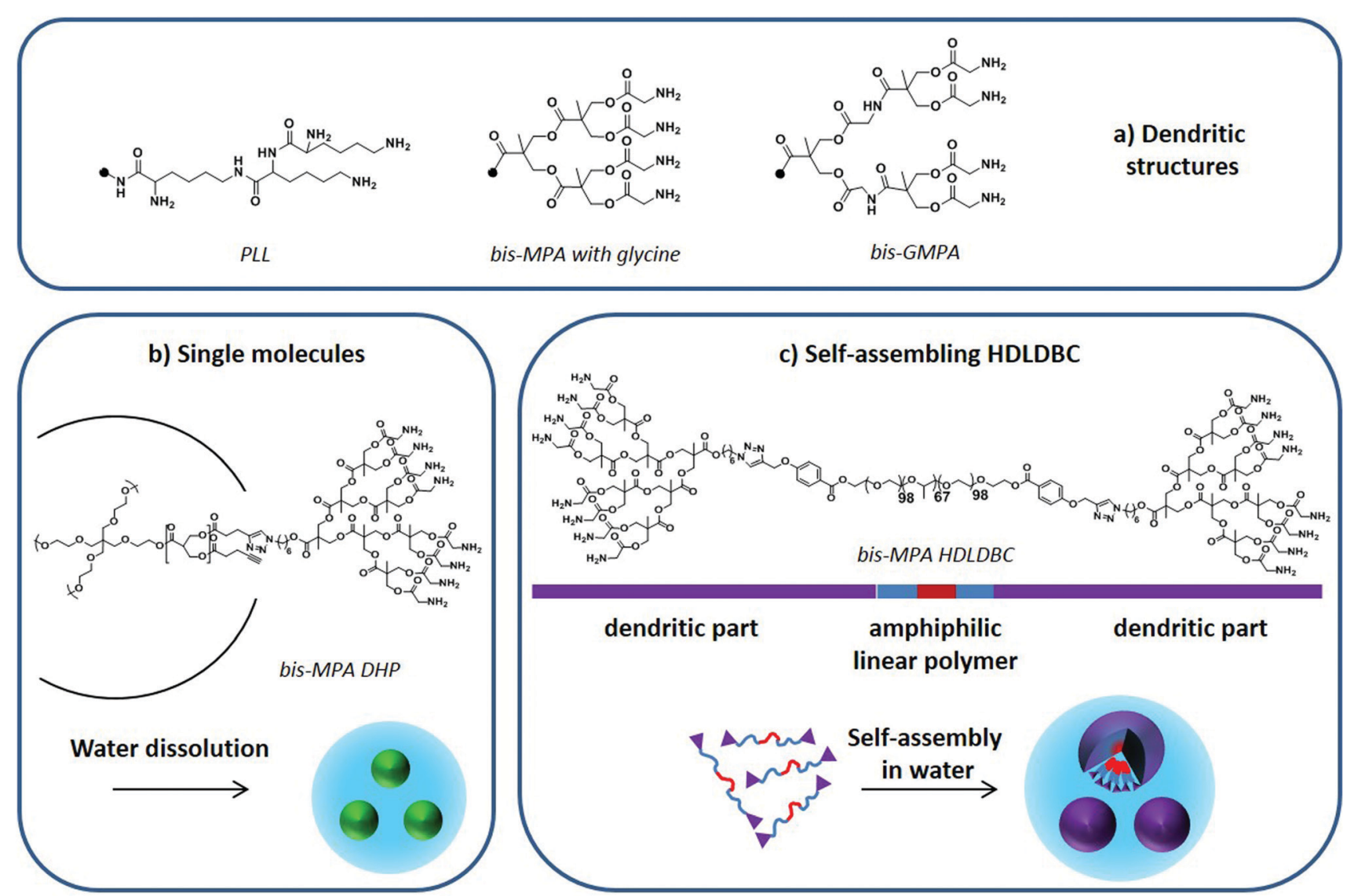

Fig. 9 (a) PLL, bis-MPA and bis-GMPA dendritic structures, (b) DHP "single molecule" dissolution in water, and (c) amphiphilic HDLDBC based on Pluronic F-127 and bis-MPA dendron self-assembly in water. Adapted from Movellan et al. ${ }^{129}$ and Martí Coma-Cros et al. ${ }^{128}$

protein isolate-carbopol-polyacrylamide-based hydrogels, which exhibited $\mathrm{pH}$ sensitivity and were biodegradable after drug release. In this case, the hydrogels were loaded with CQ diphosphate and curcumin and their release mechanism was also appropriate as dual drug delivery systems. More recently, Dawre et al. ${ }^{121}$ formulated biodegradable hydrogels formed in situ taking advantage of the rapid phase transition of lyotropic liquid crystals in an environment that mimics intracellular conditions. A mixture of oleic acid, dibutyl sebacate and polysorbate 80 composed the lyotropic liquid crystalline preconcentrates, into which the antimalarials arteether and lumefantrine were dissolved. The addition of the biodegradable copolymer PLGA allowed a higher than threefold enhancement of the hydrogel strength and sustained release of the drugs. In addition, modified in vivo Peter's four day suppressive antimalarial assay ${ }^{122}$ and a clinical simulation study revealed a rapid reduction in parasitemia levels with no recrudescence and complete mice survival when using this efficacious therapeutic system.

4.2.4 Dendrimers. Dendrimers are monodisperse hyperbranched polymers that present a high number of functional moieties on their periphery leading to multivalency. This feature, known as the "dendritic effect", can enhance the properties associated with these moieties and was particularly used as an advantage in nanomedicine. ${ }^{123}$ The unique characteristics of dendrimers have been also exploited to enhance the pharmacokinetic properties of antimalarial drugs.

In 2005, a poly-L-lysine (PLL) dendrimer (Fig. 9a) modified with PEG was first employed to improve the solubility of lipophilic artemether in water, boosting at the same time its controlled release in vitro. ${ }^{124}$ Dendrimers coated with sugars, commonly called glycodendrimers, were later used to encapsulate CQ and PQ. A galactose-coated polypropylene imine dendrimer ${ }^{125}$ and galactose-coated PLL ${ }^{126}$ could encapsulate $\mathrm{PQ}$ and CQ, respectively, enhancing their blood circulation time in vitro and in vivo. Chondroitin sulfate $\mathrm{A}^{-}$and PEG-coated PLL increased the stability of CQ in blood as well as its circulation time in vivo. ${ }^{127}$ In all cases, the addition of sugar moieties at the dendrimer's periphery led to higher drug encapsulation levels while reducing the toxicity of the nanocarriers.

Dendrimers based on a biocompatible and biodegradable 2,2-bis(hydroxymethyl)propionic acid (bis-MPA) skeleton modified with glycine moieties at their periphery (Fig. 9a) have also been used to encapsulate antimalarial drugs. Both single macromolecules, i.e. dendronized hyperbranched molecules (DHP) (Fig. 9b), ${ }^{128}$ and self-assembled amphiphilic structures, i.e. hybrid dendritic-linear-dendritic block copolymers (HDLDBC) (Fig. 9c) and Janus dendrimers, have been used. ${ }^{129}$ They all showed high drug loading capacities and excellent 
targeting towards pRBCs. Moreover, the amphiphilic HDLDBCs, which are based on linear Pluronic F-127 and bisMPA dendrons, could enhance the activity of CQ and PQ in vitro. A similar amphiphilic HDLDBC based on Pluronic F-127 and a novel dendritic structure based on poly(esteramide) 2,2-bis(glycyloxymethyl)propionic acid (bis-GMPA in Fig. 9a) has been described to encapsulate CQ, PQ and quinacrine. It displayed high drug loading capacity and good targeting towards pRBCs while showing a similar drug activity after encapsulation. ${ }^{128}$

Artemisinin has been also covalently linked to 1 st generation dendrimers leading to trimeric and tetrameric molecules. The trimeric dendrimer showed a higher drug activity in vitro than the free artesumic acid precursor and slightly lower than free dihydroartemisinin, whereas the tetrameric dendrimer exhibited a lower drug activity. The lower activity of the tetrameric dendrimer was related to its high lipophilicity, which might limit its solubility in the culture media of malaria parasites. ${ }^{130}$

\subsection{Inorganic NPs}

The research on NPs of inorganic composition as components of potential treatments for malaria infection has received increasing attention in recent years. However, it is still an area that deserves further research in order to understand the mechanism of action of different NPs and the stage of infection in which they can be effective to prevent or cure malaria. Most of the studies selected from recent years deal with NPs that have active participation in the prevention or cure of malaria. Such is the case of the metallic NPs and iron oxide NPs described below. In addition, inorganic NPs of mesoporous silica composition have been tested as effective carriers for traditional antimalarial drugs.

4.3.1 Metallic NPs. The possibilities of action of metallic NPs extend from attacking the mosquito vector to treating malaria during the human infection cycle. Most metallic NPs explored for these purposes are made of noble metals including silver, gold and palladium, as extensively reviewed in Rahman et al. ${ }^{10}$ and Barabadi et al. ${ }^{11}$ In particular, silver has shown good antibacterial properties due to the release of $\mathrm{Ag}^{+}$ions, which likely interact with thiol and phosphate groups in proteins and DNA. This interaction disrupts the cell wall integrity, impairing essential enzymes, inactivating bacterial DNA and RNA or binding subcellular components. ${ }^{131}$ The broad-spectrum antimicrobial activity of silver has also been explored against malaria, and this, together with the unique properties of its nanostructured form, has opened ways for malaria treatments based on silver NPs (AgNPs) against different stages of the infection, in both the mosquito and human hosts. ${ }^{132}$ As for the former, a crucial issue for their application is the possible ecological damage when used to replace classical synthetic insecticides. In this respect, there are many studies that indicate their innocuity for non-target aquatic microorganisms. ${ }^{132}$ As for toxicity for human hosts, the influence of the AgNP size on the biocompatibility is known. Sizes larger than $200 \mathrm{~nm}$ accumulate in the liver and spleen. Nevertheless, toxicity in humans seems to come from the release of the ionic form of silver rather than from the nanoparticulate form of silver metal, as reviewed by Rai et al. ${ }^{132}$

The activity of AgNPs is greatly influenced by their morphological and physical features such as, among others, size, shape and aggregation propensity. Although the control of these aspects during synthesis is not yet fully mastered, the benefits of biological processes to prepare AgNPs are broadly accepted. Indeed, the synthesis of metal NPs from plant extracts has become an eco-friendly and cost-effective process, and provides different types of reducing and capping agents to yield stable AgNPs. A comprehensive review by Benelli et al. gathers the biosynthesis of NPs from plant extracts, and how this type of process brings the great potential of such nanosystems to the fight against the vectors of many infectious diseases such as malaria. ${ }^{133}$ An important aspect of this research is that different plants can be classified as reducing and capping agents to prepare active metal NPs. A significant amount of work has been done in India to develop medical products from Indian medicinal plants, including nanotechnology-based ones.

Mosquitocidal activity of metallic NPs has been described at different stages of the insect's life cycle with increased pesticide efficacy compared to plant-based preparations. Thus, the half lethal concentration, LC50, of synthesized AgNPs estimated for eggs, larvae, pupae and adults has been found to be between 1 and $40 \mu \mathrm{g} \mathrm{ml} \mathrm{m}^{-1}$, which is a much lower value than that observed for the corresponding plant extracts. ${ }^{133}$ AgNPs have been mainly applied as larvicides but are also active as ovicides, adulticides, and pupicides. It is important to remark that they do not show toxicity for non-target organisms. ${ }^{134}$ Other effects of AgNPs are to reduce mosquito longevity and fecundity as well as to act as a lure and kill approach. This has been observed for AgNPs synthesized from a plant that provides capping with metabolites that are attractive for a kind of mosquito. ${ }^{135}$ In addition to $\mathrm{Ag}$, other metal compositions including $\mathrm{Au}, \mathrm{Pd}$, $\mathrm{Co}, \mathrm{Ni}, \mathrm{Cu}$ and also $\mathrm{ZnO}$ and $\mathrm{TiO}_{2}$ have shown toxicity against the mosquito larval stage, features that could be conveniently exploited to prevent malaria. ${ }^{11}$

The utilization of noble metal-based NPs as antimalarial agents has been less described. Leaf extracts of Andrographis paniculata allowed the synthesis of AgNPs with diameter around $55 \mathrm{~nm}$, which showed antiplasmodial activity. ${ }^{136}$ More recently, the same authors described the great potential of fern extracts from Indian plants to synthesize AgNPs with promising inhibitory activity against CQ-resistant $P$. falciparum. ${ }^{137}$

Similarly to AgNPs, biosynthetic procedures from leaf extracts also permitted the synthesis of PdNPs that decreased significantly (by 78\%) the parasite growth in mice infected with $P$. berghei, with a half maximal inhibitory concentration (IC50) value of $16.44 \mathrm{mg} \mathrm{Kg}{ }^{-1}$ body weight. ${ }^{138}$ With respect to the synthesis, leaf aqueous extracts of Eclipta prostrata were employed, providing a green and cheap process to prepare PdNPs stable in solution with an average size of $27 \mathrm{~nm}$ and promising antimalarial activity.

Au-N-LK3 AuNPs with sizes between 5 and $50 \mathrm{~nm}$ and prepared from the marine actinobacteria Streptomyces spp. 
delayed parasitemia by $6 \%$ at day 8 of infection, and increased survival from 50 to $85 \%$ in in vivo experiments with P. bergheiinfected mice. ${ }^{139}$ The role attributed to Au-N-LK3 is related to the response of the organism to parasite infection, helping to regulate the presence of blood factors that play an important role in the immune response to malaria infection. In particular, the development of severe malaria alters the concentration of TNF- $\alpha$ (tumor necrosis factor $\alpha$ ) and TGF- $\beta 1$ (transforming growth factor- $\beta 1$ ). In a similar way to CQ, Au-N-LK3 inhibits the production of TNF- $\alpha$, whose concentration increases in blood upon infection.

4.3.2 Iron oxide NPs. Non-toxic iron oxide NPs, IONPs, have been successfully employed as a reservoir of ferrous ions $\left(\mathrm{Fe}^{2+}\right)$, which can improve the activity of ATS. The mechanism of action of this drug relies on its endoperoxide bond, the cleavage of which is catalyzed by $\mathrm{Fe}^{2+}$. This dependence determines the specificity of ATS and artemisinin derivatives towards infected RBCs. Indeed, cleavage of endoperoxide bonds provides reactive oxygen species that cause irreversible damage to parasite DNA and proteins. Kannan et al. have recently reported promising results with $\mathrm{Fe}_{3} \mathrm{O}_{4}$ NPs coated with 2-aminoterphthalic acid, ATA-IONPs, which provide sustained release of $\mathrm{Fe}^{2+}$ ions in a $\mathrm{pH}$-dependent manner. ${ }^{140}$ Low $\mathrm{pH}$ favors the slow conversion of $\mathrm{Fe}^{3+}$ to $\mathrm{Fe}^{2+}$, which activates ATS.

Interestingly, such low $\mathrm{pH}$ is encountered in the interior of the parasite food vacuole and this favors the specificity of such ATA-IONP-fortified ATS towards Plasmodium during the trophozoite stage (Fig. 10). These results provide the possibility of overcoming problems related to artemisinin-resistant malaria parasites. Furthermore, in vivo studies in $P$. berghei-infected mice showed that a tenfold lower dose compared to free ATS was enough to decrease parasitemia by $80 \%$ on day 7 of infection. A combination of $30 \mathrm{mg} \mathrm{Kg}{ }^{-1}$ ATS and $5 \mathrm{mg} \mathrm{Kg}{ }^{-1}$ ATA-IONPs showed the same antimalarial action as $60 \mathrm{mg} \mathrm{Kg}^{-1}$ of the free drug.
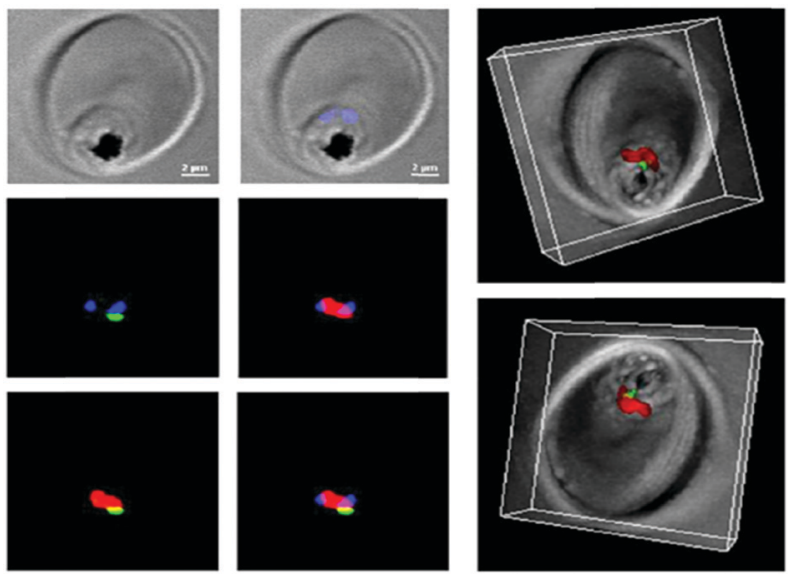

Fig. 10 Confocal microscopy was used to explore the localization of fluorescein-labeled ATA-IONPs, which appear in green within the food vacuole compartment of Plasmodium marked by LysoTracker Red. The nucleus of Plasmodium is labeled by Hoechst in blue. Figure reprinted (adapted) with permission from Kannan et al.; ${ }^{140}$ copyright (2019) Elsevier.
4.3.3 Mesoporous silica NPs. Mesoporous silica NPs (MSNs) have demonstrated good potential as nanocarriers for drug delivery due to their properties related to high loading capacity, scalable synthesis, adjustable pore size and volume, high intracellular uptake, thermal stability and excellent biocompatibility. ${ }^{141,142}$ Amolegbe et al. have studied the potential of mesoporous MCM-41 as a carrier for antimalarial drugs. ${ }^{143,144}$ MCM-41 is a silicate/aluminosilicate mesoporous molecular sieve synthesized by Beck et al. using liquid crystal templates. ${ }^{145}$ With the aim of improving bioavailability, unmodified MCM-41 and MCM-41 pore-functionalized with either amino (aMCM-41) or phenyl groups (pMCM-41) were tested to encapsulate ATS, a drug with a short half-life in blood, ${ }^{143}$ and $\mathrm{QN}$, a lipophilic drug that can cause severe adverse effects at therapeutic doses. ${ }^{144}$ The interactions between MSNs and the drug determined the release profile, whereby aMCM-41 showed slow release of ATS due to hydrogen-bonding interactions between the amino groups in the MSNs and the ATS carboxylic group. Such slow release of the drug can make these MSNs also interesting for oral administration. ${ }^{143}$ Regarding QN, the functionalization of pMCM-41 with phenyl rings appeared as a useful strategy to increase the drug loading capacity and give better controlled release. ${ }^{144}$ Both systems were tested in vitro against $P$. falciparum and in vivo against $P$. berghei-infected mice. ${ }^{144}$ The in vitro results showed the best activity for unmodified MSNs encapsulating QN, MCM-41/QN, whereas aminomodified aMCM-41/ATS was found to be inactive. However, the best in vivo results were found for both the unmodified MCM-41/QN and aMCM-41/ATS systems. MCM41/QN showed the highest effectiveness in delivering the drug to the target cells with ED50 (effective dose causing 50\% inhibition) $<0.0625 \mathrm{mg} \mathrm{Kg}^{-1}$ body weight, and an acceptable mean survival time.

\subsection{Miscellaneous}

This section includes some other recently explored molecular platforms that, although they do not fit within the previous classification, have shown interesting antimalarial properties either on their own or acting as antimalarial drug carriers and hence their use is considered promising for future malaria therapeutic strategies.

4.4.1 Graphene. The dense 2D-structure and chemical, electrical and mechanical properties of graphene and graphene oxide have fostered their application in wearable fabrics that may include functionalities such as sensing, temperature control, energy storage or water-vapor transmission, and elasticity compatible with stretchable fabrics. ${ }^{146}$ Hurt et al. have explored these properties to include graphene-based films in fabrics in order to make them resistant to mosquito biting, thus preventing infectious diseases transmitted through such vectors. ${ }^{147}$ Under dry conditions, graphene oxide (GO) and reduced graphene oxide (rGO) are protective against mosquito biting because of their molecular barrier properties that make fabrics impermeable to skin-associated molecular attractants. Under wet conditions, e.g. sweat, this molecular barrier loses effectiveness but rGO still maintains its mechanical properties, preventing mosquito puncturing. In contrast, the hydrophilic 
nature of the oxygen-containing groups in GO favors the formation of a hydrogel upon wetting, which facilitates mosquito sting penetration.

Due to its excellent biocompatibility, ${ }^{148} \mathrm{GO}$ has also been explored for its active role in preventing $\mathrm{RBC}$ infection by Plasmodium. Lim et al. have proposed a two-pronged action mechanism of GO nanosheets, which are able to inhibit significantly the invasion of RBCs by P. falciparum parasites. ${ }^{149}$ First, GO nanosheets constitute a physical barrier against parasite infection by capturing merozoites present in blood, thus impeding their adhesion to RBCs. Second, electrostatic and $\pi-\pi$ interactions impart GO nanosheets with a strong affinity for many biomolecules, among which are the nutrients required for parasite growth; their removal from blood delays the intraerythrocytic maturation of Plasmodium, and hence reduces its viability.

4.4.2 Cyclodextrin complexes. Cyclodextrins (CD) are cyclic oligosaccharides formed from glucose units that are highly biocompatible, interact with a wide range of molecules and biomolecules and are susceptible to easy functionalization to tune specific interactions. These features make them highly promising platforms for biomedical applications. ${ }^{150}$ Native CDs, namely those natural products formed by 6,7 and 8 glucoses, $\alpha-C D, \beta-C D$, and $\gamma-C D$, respectively, as well as modified CDs, some of them already approved for human use, e.g. 2-hydroxypropyl- $\beta$-cyclodextrin (2-HP- $\beta$-CD), have been explored to develop medical treatments for infectious diseases including malaria. For instance, anionic CDs with sulfate groups have proven successful to inhibit $P$. falciparum growth and to reduce parasitemia in $P$. berghei-infected mice, which correlated with the degree of sulfate functionalization. ${ }^{151}$ Nevertheless, the ability of CDs to form soluble complexes with low water-soluble molecules provides an interesting strategy to investigate novel treatments against malaria based on the improved oral bioavailability of already known lipophilic antimalarials. ${ }^{4}$ Microparticles of artemisinin/ $\beta$-CD complexes formed by spray drying provided agglomerates suitable for oral administration. The interaction with $\beta$-CD increased six-fold the solubility of artemisinin relative to the free drug, and hence its oral bioavailability, as demonstrated in in vivo experiments in rats. ${ }^{152}$ Unlike artemisinin, for which the formation of inclusion drug/CD complexes was not fully confirmed, and depended on the drug/ $\beta-\mathrm{CD}$ ratio, ${ }^{152}$ artemether has been reported to form stable inclusion complexes with 2-HP- $\beta$-CD with high entrapment efficiency, which increased its solubility. ${ }^{153}$ In vivo studies in rats showed that the intragastric administration of the complex resulted in faster blood absorption and bioavailability than for the free drug.

\section{Future perspectives and conclusions}

After one hundred years of using classical therapeutic (drugs) and prophylactic (vaccines) approaches that have not succeeded in eradicating malaria, nanotechnology and nanomaterials can offer new opportunities to build customized tools for prophylactic and therapeutic purposes that bring new solutions in different stages of malaria pathophysiology.
Issues concerning drug solubility, biostability, toxicity and uncontrolled pharmacokinetics can be overcome with adequately designed drug NCs based on liposomes, polymers or dendrimers. Furthermore, drug resistance evolves when one cannot deliver to the parasites a sufficiently high dose to kill them rapidly. This happens more easily when the drug is not specifically targeted to the pathogen and therefore it distributes throughout the body, which imposes a maximum overall dose that does not trigger undesirable side effects. NCs carrying binding molecules for specific receptors present only on cells infected by the malaria parasite, represent a highly promising option.

For most malaria cases, to be treated in endemic regions with poor or inexistent healthcare infrastructure, and in order to favor patient compliance, oral delivery will be the preferred option and polymer-based NCs can be made apt for this. However, for severe malaria cases where the patient is usually hospitalized, or in other contexts such as in a future malaria eradication campaign where the disease will be entrenched in small contained areas, intravenous administration can be reasonably considered. In these cases, liposomal NCs are an interesting alternative since they have a long track record of successful clinical applications in a wide variety of diseases.

Targeting specificity to infected cells is a key issue that can be also faced with NCs. Since they can operate at the molecular level, nanomaterials can be more precise in reaching a particular drug target, or they can be designed to present several antigens to immune cells for the production of a new generation of better vaccines.

Another possibility for prevention is attacking the mosquito vector. In this respect, mainly metal-based NPs but also liposome-based ones have been shown to be successful to prevent malaria infection.

The main concern when using nanomaterials is related to their production cost, which is an issue to consider when dealing with pathologies like malaria, where the vast majority of cases are in poverty-ridden areas. Pathologies that are not prominent in high income regions have little chance of attracting industrial interest since the large investment in product development will not be recovered as easily as when selling drugs to treat carcinomas, Alzheimer's disease or obesity. However, severe malaria is such a serious life-threatening condition and eradication is such a long sought-after goal that in these cases arguing economic reasons would be out of place. Examples of ways to overcome this obstacle exist, such as the current commitment of a few big pharma companies to devote part of their budget to infectious disease research, but more public and private economic efforts are needed. Some of the basic research results obtained in this field are highly promising, but it is necessary to capture the interest of clinicians and establish collaborations with the medical sector in order to adapt this new technology to the requirements of its deployment as a treatment for people.

\section{Conflicts of interest}

The authors declare no conflicts of interest. 


\section{Acknowledgements}

This research was supported by grants RTI2018-094579-B-I00, PCIN-2017-100, PGC2018-097583-B-I00, PGC2018-093761-B-C31, CTQ2017-90596-REDT (Ministerio de Ciencia, Innovación y Universidades, Spain, which included FEDER funds), 2017-178 (ERA-NET Cofund EURONANOMED), 2017-SGR-908 (Generalitat de Catalunya, Spain), and E47_20R (Gobierno de Aragón-FSE). ISGlobal and IBEC are members of the CERCA Programme, Generalitat de Catalunya, and both acknowledge support from the Spanish Ministry of Science, Innovation and Universities through the "Centro de Excelencia Severo Ochoa 2019-2023" Program (CEX2018-000806-S). This research is part of ISGlobal's Program on the Molecular Mechanisms of Malaria, which is partially supported by the Fundación Ramón Areces. LNB-C was supported by the European Commission under Horizon 2020's Marie Skłodowska-Curie Actions COFUND scheme (712754) and by the Severo Ochoa programme of the Spanish Ministry of Science and Competitiveness, SEV-2014-0425 (2015-2019). MSA thanks the Ministerio de Ciencia, Innovación y Universidades, for her grant (BES-2016-078774). We acknowledge support of the publication fee by the CSIC Open Access Publication Support Initiative through its Unit of Information Resources for Research (URICI).

\section{References}

1 TDR Strategy 2018-2023, https://www.who.int/tdr/publica tions/about-tdr/strategy/strategy-2018-23/en/.

2 World Malaria Report 2019. Accessed May 2020, https://www. who.int/publications-detail/world-malaria-report-2019.

3 G. A. Islan, M. Durán, M. L. Cacicedo, G. Nakazato, R. K. T. Kobayashi, D. S. T. Martinez, G. R. Castro and N. Durán, Nanopharmaceuticals as a solution to neglected diseases: is it possible?, Acta Trop., 2017, 170, 16-42.

4 N. S. Santos-Magalhães and V. C. F. Mosqueira, Nanotechnology applied to the treatment of malaria, Adv. Drug Delivery Rev., 2010, 62, 560-575.

5 P. Urbán and X. Fernàndez-Busquets, Nanomedicine against malaria, Curr. Med. Chem., 2014, 21, 605-629.

6 E. Dennis, V. A. Peoples, F. Johnson, R. K. Bibbs, D. Topps, A. Bopda-Waffo and M. T. Coats, Utilizing nanotechnology to combat malaria, J. Infect. Dis. Ther., 2015, 3, 229.

7 S. Tripathy and S. Roy, A review of age-old antimalarial drug to combat malaria: efficacy up-gradation by nanotechnology based drug delivery, Asian Pac. J. Trop. Med., 2014, 7, 673-679.

8 N. Puttappa, R. S. Kumar, G. Kuppusamy and A. Radhakrishnan, Nano-facilitated drug delivery strategies in the treatment of Plasmodium infection, Acta Trop., 2019, 195, 103-114.

9 R. S. Oakes and C. M. Jewell, A plug-and-play approach for malaria vaccination, Nat. Nanotechnol., 2018, 13, 1096-1097.

10 K. Rahman, S. U. Khan, S. Fahad, M. X. Chang, A. Abbas, W. U. Khan, L. Rahman, Z. Ul Haq, G. Nabi and D. Khan,
Nano-biotechnology: a new approach to treat and prevent malaria, Int. J. Nanomed., 2019, 14, 1401-1410.

11 H. Barabadi, Z. Alizadeh, M. T. Rahimi, A. Barac, A. E. Maraolo, L. J. Robertson, A. Masjedi, F. Shahrivar and E. Ahmadpour, Nanobiotechnology as an emerging approach to combat malaria: a systematic review, Nanomedicine, 2019, 18, 221-233.

12 R. Carter and K. N. Mendis, Evolutionary and historical aspects of the burden of malaria, Clin. Microbiol. Rev., 2002, 15, 564-594.

13 A. Amir, F. W. Cheong, J. R. De Silva, J. W. K. Liew and Y. L. Lau, Plasmodium knowlesi malaria: current research perspectives, Infect. Drug Resist., 2018, 1145-1155.

14 G. E. Weiss, B. S. Crabb and P. R. Gilson, Overlaying molecular and temporal aspects of malaria parasite invasion, Trends Parasitol., 2016, 32, 284-295.

15 C. R. S. Garcia, R. P. Markus and L. Madeira, Tertian and quartan fevers: temporal regulation in malarial infection, J. Biol. Rhythms, 2001, 16, 436-443.

16 J. D. Smith, J. A. Rowe, M. K. Higgins and T. Lavstsen, Malaria's deadly grip: cytoadhesion of Plasmodium falciparum-infected erythrocytes, Cell. Microbiol., 2013, 15, 1976-1983.

17 A. Rowe, J. Obeiro, C. I. Newbold and K. Marsh, Plasmodium falciparum rosetting is associated with malaria severity in Kenya, Infect. Immun., 1995, 63, 2323-2326.

18 A. Muthusamy, R. N. Achur, V. P. Bhavanandan, G. G. Fouda, D. W. Taylor and D. C. Gowda, Plasmodium falciparum-infected erythrocytes adhere both in the intervillous space and on the villous surface of human placenta by binding to the low-sulfated chondroitin sulfate proteoglycan receptor., Am. J. Pathol., 2004, 164, 2013-2025.

19 G. A. Josling, K. C. Williamson and M. Llinás, Regulation of sexual commitment and gametocytogenesis in malaria parasites, Annu. Rev. Microbiol., 2018, 72, 501-519.

20 World Health Organization, Guidelines for the Treatment of Malaria, 3rd edn, WHO Press, Geneva, Switzerland, 2015.

21 E. G. Tse, M. Korsik and M. H. Todd, The past, present and future of anti-malarial medicines, Malar. J., 2019, 18, 93.

22 J. Achan, A. O. Talisuna, A. Erhart, A. Yeka, J. K. Tibenderana, F. N. Baliraine, P. J. Rosenthal and U. D'Alessandro, Quinine, an old anti-malarial drug in a modern world: role in the treatment of malaria, Malar. J., 2011, 10, 144.

23 S. J. Rogerson, Management of malaria in pregnancy, Indian J. Med. Res., 2017, 146, 328-333.

24 S. Mvango, W. M. R. Matshe, A. O. Balogun, L. A. Pilcher and M. O. Balogun, Nanomedicines for malaria chemotherapy: encapsulation vs. polymer therapeutics, Pharm. Res., 2018, 35, 237.

25 C. Nsanzabana, Resistance to artemisinin combination therapies (ACTs): do not forget the partner Drug!, Trop. Med. Infect. Dis., 2019, 4, 26.

26 J. Marques, E. Moles, P. Urbán, R. Prohens, M. A. Busquets, C. Sevrin, C. Grandfils and X. Fernàndez-Busquets, Application of heparin as a dual agent with antimalarial and liposome targeting activities toward Plasmodium-infected red blood cells, Nanomedicine, 2014, 10, 1719-1728. 
27 M. A. Ali, M. A. Penny, T. A. Smith, L. Workman, P. Sasi, G. O. Adjei, F. Aweeka, J.-R. Kiechel, V. Jullien, M. J. Rijken, R. McGready, J. Mwesigwa, K. Kristensen, K. Stepniewska, J. Tarning, K. I. Barnes and P. Denti, Population pharmacokinetics of the antimalarial amodiaquine: a pooled analysis to optimize dosing, Antimicrob. Agents Chemother., 2018, 62, e02193.

28 V. Navaratnam, S. M. Mansor, N.-W. Sit, J. Grace, Q. Li and P. Olliaro, Pharmacokinetics of artemisinin-type compounds, Clin. Pharmacokinet., 2000, 39, 255-270.

29 P. Newton, Y. Suputtamongkol, P. Teja-Isavadharm, S. Pukrittayakamee, V. Navaratnam, I. Bates and N. White, Antimalarial bioavailability and disposition of artesunate in acute falciparum malaria, Antimicrob. Agents Chemother., 2000, 44, 972-977.

30 J. L. Das, S. Rulisa, P. J. De Vries, P. F. Mens, N. Kaligirwa, S. Agaba, J. Tarning, M. O. Karlsson and T. P. C. Dorlo, Population pharmacokinetics of artemether, dihydroartemisinin, and lumefantrine in Rwandese pregnant women treated for uncomplicated Plasmodium falciparum malaria, Antimicrob. Agents Chemother., 2018, 62, e00518.

31 H. Liu, H. Zhou, T. Cai, A. Yang, M. Zang and J. Xing, Metabolism of piperaquine to its antiplasmodial metabolites and their pharmacokinetic profiles in healthy volunteers, Antimicrob. Agents Chemother., 2018, 62, e00260.

32 S. Krishna and N. J. White, Pharmacokinetics of quinine, chloroquine and amodiaquine: clinical implications, Clin. Pharmacokinet., 1996, 30, 263-299.

33 J.-F. O. Trape, The public health impact of chloroquine resistance in Africa, Am. J. Trop. Med. Hygene, 2001, 64, 12-17.

34 N. J. White, Sulfadoxine-pyrimethamine for the treatment of malaria, Trans. R. Soc. Trop. Med. Hyg., 1991, 85, 556-557.

35 D. M. Harmening, V. C. Hughes and C. Del Toro, Clinical Hematology and Fundamentals of Hemostasis, F. A. Davis Company, Philadelphia, 1997, pp. 54-70.

36 C. H. Villa, A. C. Anselmo, S. Mitragotri and V. Muzykantov, Red blood cells: supercarriers for drugs, biologicals, and nanoparticles and inspiration for advanced delivery systems, Adv. Drug Delivery Rev., 2016, 106, 88-103.

37 T. Dai, W. Jiang, Z. Guo, Y. Xie and R. Dai, Comparison of in vitro/in vivo blood distribution and pharmacokinetics of artemisinin, artemether and dihydroartemisinin in rats, J. Pharm. Biomed. Anal., 2019, 162, 140-148.

38 D. W. Wilson, C. Langer, C. D. Goodman, G. I. McFadden and J. G. Beeson, Defining the timing of action of antimalarial drugs against Plasmodium falciparum, Antimicrob. Agents Chemother., 2013, 57, 1455-1467.

39 E. Moles, P. Urbán, M. B. Jiménez-Díaz, S. Viera-Morilla, I. Angulo-Barturen, M. A. Busquets and X. FernàndezBusquets, Immunoliposome-mediated drug delivery to Plasmodium-infected and non-infected red blood cells as a dual therapeutic/prophylactic antimalarial strategy, J. Controlled Release, 2015, 210, 217-229.

40 E. Moles and X. Fernàndez-Busquets, Loading antimalarial drugs into noninfected red blood cells: an undesirable roommate for Plasmodium, Future Med. Chem., 2015, 7, 837-840.
$41 \mathrm{~K}$. Kirk, Membrane transport in the malaria-infected erythrocyte, Physiol. Rev., 2001, 81, 495-537.

42 H. P. De Koning, A. Kaddoumi, P. Hsiao, S. A. Desai, K. Basore, Y. Cheng, A. K. Kushwaha and S. T. Nguyen, How do antimalarial drugs reach their intracellular targets?, Front. Pharmacol., 2015, 6, 1-7.

43 P. Urbán, J. Estelrich, A. Cortés and X. FernàndezBusquets, A nanovector with complete discrimination for targeted delivery to Plasmodium falciparum-infected versus non-infected red blood cells in vitro, J. Control. Release, 2011, 151, 202-211.

44 G. M. Hasan, N. Garg, E. Dogra, R. Surolia and P. C. Ghosh, Inhibition of the growth of Plasmodium falciparum in culture by stearylamine-phosphatidylcholine liposomes, J. Parasitol. Res., 2011, 2011, 1-9.

45 A. D. Pillai, W. Nguitragool, B. Lyko, K. Dolinta, M. M. Butler, S. T. Nguyen, N. P. Peet, T. L. Bowlin and S. A. Desai, Solute restriction reveals an essential role for clag3-associated channels in malaria parasite nutrient acquisition, Mol. Pharmacol., 2012, 82, 1104-1114.

46 A. Alkhalil, J. V. Cohn, M. A. Wagner, J. S. Cabrera, T. Rajapandi and S. A. Desai, Plasmodium falciparum likely encodes the principal anion channel on infected human erythrocytes, Blood, 2004, 104, 4279-4286.

47 A. Alkhalil, A. D. Pillai, A. A. B. Bokhari, A. B. Vaidya and S. A. Desai, Complex inheritance of the plasmodial surface anion channel in a Plasmodium falciparum genetic cross, Mol. Microbiol., 2009, 72, 459-469.

48 S. Mira-Martínez, N. Rovira-Graells, V. M. Crowley, L. M. Altenhofen, M. Llinás and A. Cortés, Epigenetic switches in clag3 genes mediate blasticidin S resistance in malaria parasites, Cell. Microbiol., 2013, 15, 1913-1923.

49 R. E. Martin, R. V. Marchetti, A. I. Cowan, S. M. Howitt, S. Bröer and K. Kirk, Chloroquine transport via the malaria parasite's chloroquine resistance transporter, Science, 2009, 325, 1680-1682.

50 K. Bachhawat, C. J. Thomas, N. Surolia and A. Surolia, Interaction of chloroquine and its analogues with heme: an isothermal titration calorimetric study, Biochem. Biophys. Res. Commun., 2000, 276, 1075-1079.

51 M. Henry, S. Briolant, A. Zettor, S. Pelleau, M. Baragatti, E. Baret, J. Mosnier, R. Amalvict, T. Fusai, C. Rogier and B. Pradines, Plasmodium falciparum $\mathrm{Na}^{+} / \mathrm{H}^{+}$exchanger 1 transporter is involved in reduced susceptibility to quinine, Antimicrob. Agents Chemother., 2009, 53, 1926-1930.

52 R. N. Price, A.-C. Uhlemann, A. Brockman, R. Mcgready, E. Ashley, L. Phaipun, R. Patel, K. Laing, S. Looareesuwan, N. J. White, F. Nosten and S. Krishna, Mefloquine resistance in Plasmodium falciparum and increased pfmdr1 gene copy number, Lancet, 2004, 364, 438-447.

53 A. B. S. Sidhu, A. Uhlemann, S. G. Valderramos, J. Valderramos, S. Krishna and D. A. Fidock, Decreasing pfmdr1 copy number in Plasmodium falciparum malaria heightens susceptibility to mefloquine, lumefantrine, halofantrine, quinine, and artemisinin, J. Infect. Dis., 2006, 194, 528-535. 
54 R. S. Lee, A. P. Waters and J. M. Brewer, A cryptic cycle in haematopoietic niches promotes initiation of malaria transmission and evasion of chemotherapy, Nat. Commun., 2018, 9, 1-9.

55 G. Gregoriadis, Drug entrapment in liposomes, FEBS Lett., 1973, 36, 292-296.

56 A. S. Abu Lila and T. Ishida, Liposomal delivery systems: design optimization and current applications, Biol. Pharm. Bull., 2017, 40, 1-10.

57 R. Nisini, N. Poerio, S. Mariotti, F. De Santis and M. Fraziano, The multirole of liposomes in therapy and prevention of infectious diseases, Front. Immunol., 2018, 9, 155.

58 A. Akbarzadeh, R. Rezaei-Sadabady, S. Davaran, S. W. Joo, N. Zarghami, Y. Hanifehpour, M. Samiei, M. Kouhi and K. Nejati-Koshki, Liposome: classification, preparation, and applications, Nanoscale Res. Lett., 2013, 8, 102.

59 M. S. Mufamadi, V. Pillay, Y. E. Choonara, L. C. Du Toit, G. Modi, D. Naidoo and V. M. K. Ndesendo, A review on composite liposomal technologies for specialized drug delivery, J. Drug Delivery, 2011, 2011, 939851.

60 N. P. Aditya, G. Chimote, K. Gunalan, R. Banerjee, S. Patankar and B. Madhusudhan, Curcuminoids-loaded liposomes in combination with arteether protects against Plasmodium berghei infection in mice, Exp. Parasitol., 2012, 131, 292-299.

61 N. Maurer, D. B. Fenske and P. R. Cullis, Developments in liposomal drug delivery systems, Expert Opin. Biol. Ther., 2001, 1, 923-947.

62 F. S. Nas, A. Yahaya and M. Ali, Application of liposomes nanoparticles in the treatment of malaria: a mini review, J. Biotechnol. Biores., 2018, 1, JBB.000510.

63 H. He, Y. Lu, J. Qi, Q. Zhu, Z. Chen and W. Wu, Adapting liposomes for oral drug delivery, Acta Pharm. Sin. B, 2019, 9, 36-48.

64 E. M. Coma-Cros, A. Biosca, E. Lantero, M. L. Manca, C. Caddeo, L. Gutiérrez, M. Ramírez, L. N. BorghetiCardoso, M. Manconi and X. Fernàndez-Busquets, Antimalarial activity of orally administered curcumin incorporated in Eudragit ${ }^{\mathbb{R}}$-containing liposomes, Int. J. Mol. Sci., 2018, 19, 131.

65 C. R. Alving, Liposomes as drug carriers in leishmaniasis and malaria, Parasitol. Today, 1986, 2, 101-107.

66 P. Pirson, R. F. Steiger, A. Trouet, J. Gillet and F. Herman, Primaquine liposomes in the chemotherapy of experimental murine malaria, Ann. Trop. Med. Parasitol., 1980, 74, 383-391.

67 P. Pirson, R. Steiger and A. Trouet, The disposition of free and liposomally encapsulated antimalarial primaquine in mice, Biochem. Pharmacol., 1982, 31, 3501-3507.

68 A. Trouet, P. Pirson, R. Steiger, M. Masquelier, R. Baurain and J. Gillet, Development of new derivatives of primaquine by association with lysosomotropic carriers, Bull. W. H. O., 1981, 59, 449-458.

69 C. R. Alving, I. Schneider, G. M. Swartz and E. A. Steck, Sporozoite-induced malaria: therapeutic effects of glycolipids in liposomes, Science, 1979, 205, 1142-1144.
70 C. R. Alving, Therapeutic potential of liposomes as carriers in leishmaniasis, malaria, and vaccines, in Targeting of Drugs, ed. G. Gregoriadis, J. Senior and A. Trouet, Plenum Press, New York, 1982, pp. 337-353.

71 K. J. Longmuir, R. T. Robertson, S. M. Haynes, J. L. Baratta and A. J. Waring, Effective targeting of liposomes to liver and hepatocytes in vivo by incorporation of a Plasmodium amino acid sequence, Pharm. Res., 2006, 23, 759-769.

72 A. Pujol, P. Urbán, C. Riera, R. Fisa, I. Molina, F. Salvador, J. Estelrich and X. Fernàndez-Busquets, Application of quantum dots to the study of liposome targeting in leishmaniasis and malaria, Int. J. Theor. Appl. Nanotechnol., 2014, 2, 1-8.

73 M. Owais, G. C. Varshney, A. Choudhury, S. Chandra and C. M. Gupta, Chloroquine encapsulated in malariainfected erythrocyte-specific antibody-bearing liposomes effectively controls chloroquine-resistant Plasmodium berghei infections in mice, Antimicrob. Agents Chemother., 1995, 39, 180-184.

74 P. Urbán, J. Estelrich, A. Adeva, A. Cortés and X. FernàndezBusquets, Study of the efficacy of antimalarial drugs delivered inside targeted immunoliposomal nanovectors, Nanoscale Res. Lett., 2011, 6, 620.

75 E. Moles, S. Galiano, A. Gomes, M. Quiliano, C. Teixeira, I. Aldana, P. Gomes and X. Fernàndez-Busquets, ImmunoPEGliposomes for the targeted delivery of novel lipophilic drugs to red blood cells in a falciparum malaria murine model, Biomaterials, 2017, 145, 178-191.

76 E. Moles, K. Moll, J. H. Ch'ng, P. Parini, M. Wahlgren and $\mathrm{X}$. Fernàndez-Busquets, Development of drug-loaded immunoliposomes for the selective targeting and elimination of rosetting Plasmodium falciparum-infected red blood cells, J. Controlled Release, 2016, 241, 57-67.

$77 \mathrm{X}$. Fernàndez-Busquets, Toy kit against malaria: magic bullets, LEGO, Trojan horses and Russian dolls, Ther. Delivery, 2014, 5, 1049-1052.

78 I. L. Lisovskaya, I. M. Shcherbachenko, R. I. Volkova and F. I. Ataullakhanov, Clotrimazole enhances lysis of human erythrocytes induced by t-BHP, Chem. - Biol. Interact., 2009, 180, 433-439.

79 A. Biosca, L. Dirscherl, E. Moles, S. Imperial and X. FernàndezBusquets, An ImmunoPEGliposome for targeted antimalarial combination therapy at the nanoscale, Pharmaceutics, 2019, 11, 341.

80 V. Rajendran, S. Rohra, M. Raza, G. M. Hasan, S. Dutt and P. C. Ghosh, Stearylamine liposomal delivery of monensin in combination with free artemisinin eliminates blood stages of Plasmodium falciparum in culture and P. berghei infection in murine malaria, Antimicrob. Agents Chemother., 2016, 60, 1304-1318.

81 B. Isacchi, S. Arrigucci, G. La Marca, M. C. Bergonzi, M. G. Vannucchi, A. Novelli and A. R. Bilia, Conventional and long-circulating liposomes of artemisinin: preparation, characterization, and pharmacokinetic profile in mice, J. Liposome Res., 2011, 21, 237-244.

82 M. Ismail, L. Ling, Y. Du, C. Yao and X. Li, Liposomes of dimeric artesunate phospholipid: a combination of 
dimerization and self-assembly to combat malaria, Biomaterials, 2018, 163, 76-87.

83 S. Ibrahim, T. Tagami and T. Ozeki, Effective-loading of platinum-chloroquine into PEGylated neutral and cationic liposomes as a drug delivery system for resistant malaria parasites, Biol. Pharm. Bull., 2017, 40, 815-823.

84 J. Marques, J. J. Valle-Delgado, P. Urbán, E. Baró, R. Prohens, A. Mayor, P. Cisteró, M. Delves, R. E. Sinden, C. Grandfils, J. L. de Paz, J. A. García-Salcedo and $\mathrm{X}$. Fernàndez-Busquets, Adaptation of targeted nanocarriers to changing requirements in antimalarial drug delivery, Nanomedicine, 2017, 13, 515-525.

85 A. Barragan, D. Spillmann, P. G. Kremsner, M. Wahlgren and J. Carlson, Plasmodium falciparum: molecular background to strain-specific rosette disruption by glycosaminoglycans and sulfated glycoconjugates, Exp. Parasitol., 1999, 91, 133-143.

86 S. Glushakova, B. L. Busse, M. Garten, J. R. Beck, R. M. Fairhurst, D. E. Goldberg and J. Zimmerberg, Exploitation of a newly-identified entry pathway into the malaria parasite-infected erythrocyte to inhibit parasite egress, Sci. Rep., 2017, 7, 12250.

87 M. J. Boyle, J. S. Richards, P. R. Gilson, W. Chai and J. G. Beeson, Interactions with heparin-like molecules during erythrocyte invasion by Plasmodium falciparum merozoites, Blood, 2010, 115, 4559-4568.

88 A. M. Vogt, A. Barragan, Q. Chen, F. Kironde, D. Spillmann and M. Wahlgren, Heparan sulfate on endothelial cells mediates the binding of Plasmodium falciparum-infected erythrocytes via the $\alpha$ DBL1 domain of PfEMP1, Blood, 2003, 101, 2405-2411.

89 A. M. Vogt, G. Winter, M. Wahlgren and D. Spillmann, Heparan sulphate identified on human erythrocytes: a Plasmodium falciparum receptor, Biochem. J., 2004, 381, 593-597.

90 A. Salanti, M. Dahlbäck, L. Turner, M. A. Nielsen, L. Barfod, P. Magistrado, A. T. R. Jensen, T. Lavstsen, M. F. Ofori, K. Marsh, L. Hviid and T. G. Theander, Evidence for the involvement of VAR2CSA in pregnancyassociated malaria, J. Exp. Med., 2004, 200, 1197-1203.

91 D. J. A. Crommelin, P. van Hoogevest and G. Storm, The role of liposomes in clinical nanomedicine development. What now? Now what?, J. Controlled Release, 2020, 318, 256-263.

92 P. B. Memvanga, R. Coco and V. Préat, An oral malaria therapy: curcumin-loaded lipid-based drug delivery systems combined with $\beta$-arteether, J. Controlled Release, 2013, 172, 904-913.

93 L. Sercombe, T. Veerati, F. Moheimani, S. Y. Wu, A. K. Sood and S. Hua, Advances and challenges of liposome assisted drug delivery, Front. Pharmacol., 2015, 6, 286.

94 Z. Mhlwatika and B. A. Aderibigbe, Polymeric nanocarriers for the delivery of antimalarials, Molecules, 2018, 23, 2527.

95 P. Urbán, J. J. Valle-Delgado, N. Mauro, J. Marques, A. Manfredi, M. Rottmann, E. Ranucci, P. Ferruti and $\mathrm{X}$. Fernàndez-Busquets, Use of poly(amidoamine) drug conjugates for the delivery of antimalarials to Plasmodium, J. Controlled Release, 2014, 177, 84-95.

96 E. M. Coma-Cros, A. Biosca, J. Marques, L. Carol, P. Urbán, D. Berenguer, M. C. Riera, M. Delves, R. E. Sinden, J. J. Valle-Delgado, L. Spanos, I. Siden-Kiamos, P. Pérez, K. Paaijmans, M. Rottmann, A. Manfredi, P. Ferruti, E. Ranucci and X. Fernàndez-Busquets, Polyamidoamine nanoparticles for the oral administration of antimalarial drugs, Pharmaceutics, 2018, 10, 225.

97 P. Urbán, E. Ranucci and X. Fernàndez-Busquets, Polyamidoamine nanoparticles as nanocarriers for the drug delivery to malaria parasite stages in the mosquito vector, Nanomedicine, 2015, 10, 3401-3414.

98 S. Alven and B. Aderibigbe, Combination therapy strategies for the treatment of malaria, Molecules, 2019, 24, 3601.

99 N. Jawahar, U. K. Baruah and V. Singh, Co-delivery of chloroquine phosphate and azithromycin nanoparticles to overcome drug resistance in malaria through intracellular targeting, J. Pharm. Sci. Res., 2019, 11, 33-40.

100 P. J. Rosenthal, Azithromycin for malaria?, Am. J. Trop. Med. Hyg., 2016, 95, 2-4.

101 O. Oyeyemi, O. Morenkeji, F. Afolayan, K. Dauda, Z. Busari, J. Meena and A. Panda, Curcumin-artesunate based polymeric nanoparticle; antiplasmodial and toxicological evaluation in murine model, Front. Pharmacol., 2018, 9, 562.

102 S. E. Haas, C. C. Bettoni, L. K. de Oliveira, S. S. Guterres and T. Dalla Costa, Nanoencapsulation increases quinine antimalarial efficacy against Plasmodium berghei in vivo, Int. J. Antimicrob. Agents, 2009, 34, 156-161.

103 E. A. Leite, A. Grabe-Guimarães, H. N. Guimarães, G. L. L. Machado-Coelho, G. Barratt and V. C. F. Mosqueira, Cardiotoxicity reduction induced by halofantrine entrapped in nanocapsule devices, Life Sci., 2007, 80, 1327-1334.

104 K. Velasques, T. R. Maciel, A. H. de Castro Dal Forno, F. E. G. Teixeira, A. L. da Fonseca, F. de P. Varotti, A. R. Fajardo, D. S. de Ávila and S. E. Haas, Co-nanoencapsulation of antimalarial drugs increases their in vitro efficacy against Plasmodium falciparum and decreases their toxicity to Caenorhabditis elegans, Eur. J. Pharm. Sci., 2018, 118, 1-12.

105 H. D. Lu, K. D. Ristroph, E. L. K. Dobrijevic, J. Feng, S. A. McManus, Y. Zhang, W. D. Mulhearn, H. Ramachandruni, A. Patel and R. K. Prud'homme, Encapsulation of OZ439 into nanoparticles for supersaturated drug release in oral malaria therapy, ACS Infect. Dis., 2018, 4, 970-979.

106 F. Föger, W. Noonpakdee, B. Loretz, S. Joojuntr, W. Salvenmoser, M. Thaler and A. Bernkop-Schnürch, Inhibition of malarial topoisomerase II in Plasmodium falciparum by antisense nanoparticles, Int. J. Pharm., 2006, 319, 139-146.

107 D. Xiao, B. Yang, X. M. Yang, D. Yi, X. L. Liao, J. Yang and C. Z. Gao, Synthesis of water soluble chitosan-artemisinin conjugate, Asian J. Chem., 2013, 25, 4637-4639.

108 H. Ringsdorf, Structure and properties of pharmacologically active polymers, J. Polym. Sci., Polym. Symp., 1975, 51, 135-153.

109 J. Golenser, S. Frankenburg, T. Ehrenfreund and A. J. Domb, Efficacious treatment of experimental leishmaniasis with 
amphotericin B-arabinogalactan water-soluble derivatives, Antimicrob. Agents Chemother., 1999, 43, 2209-2214.

110 S. Nicoletti, K. Seifert and I. H. Gilbert, $N-(2-$ hydroxypropyl)methacrylamide-amphotericin B (HPMAAmB) copolymer conjugates as antileishmanial agents, Int. J. Antimicrob. Agents, 2009, 33, 441-448.

111 J. Hofsteenge, A. Capuano, R. Altszuler and S. Moore, Carrierlinked primaquine in the chemotherapy of malaria, J. Med. Chem., 1986, 29, 1765-1769.

112 N. Tomiya, J. G. Jardim, J. Hou, R. Pastrana-Mena, R. R. Dinglasan and Y. C. Lee, Liver-targeting of primaquine(poly- $\gamma$-glutamic acid) and its degradation in rat hepatocytes, Bioorg. Med. Chem., 2013, 21, 5275-5281.

113 S. Kumar, R. K. Singh, R. S. R. Murthy and T. R. Bhardwaj, Synthesis and evaluation of substituted poly(organophosphazenes) as a novel nanocarrier system for combined antimalarial therapy of primaquine and dihydroartemisinin, Pharm. Res., 2015, 32, 2736-2752.

114 S. Kumar, R. K. Singh, R. Sharma, R. S. R. Murthy and T. R. Bhardwaj, Design, synthesis and evaluation of antimalarial potential of polyphosphazene linked combination therapy of primaquine and dihydroartemisinin, Eur. J. Pharm. Sci., 2015, 66, 123-137.

115 R. P. Bakshi, L. M. Tatham, A. C. Savage, A. K. Tripathi, G. Mlambo, M. M. Ippolito, E. Nenortas, S. P. Rannard, A. Owen and T. A. Shapiro, Long-acting injectable atovaquone nanomedicines for malaria prophylaxis, Nat. Commun., 2018, 9, 315.

116 A. Najer, D. Wu, A. Bieri, F. Brand, C. G. Palivan, H.-P. Beck and W. Meier, Nanomimics of host cell membranes block invasion and expose invasive malaria parasites, ACS Nano, 2014, 8, 12560-12571.

117 A. Najer, S. Thamboo, J. T. Duskey, C. G. Palivan, H.-P. Beck and W. Meier, Analysis of molecular parameters determining the antimalarial activity of polymer-based nanomimics, Macromol. Rapid Commun., 2015, 36, 1923-1928.

118 J. Kopeček, Hydrogel biomaterials: a smart future?, Biomaterials, 2007, 28, 5185-5192.

119 B. Aderibigbe, E. Sadiku, J. Jayaramudu and S. S. Ray, Controlled dual release study of curcumin and a 4-aminoquinoline analog from gum acacia containing hydrogels, J. Appl. Polym. Sci., 2015, 132, 41613.

120 B. A. Aderibigbe and Z. Mhlwatika, Dual release kinetics of antimalarials from soy protein isolate-carbopol-polyacrylamide based hydrogels, J. Appl. Polym. Sci., 2016, 133, 43918.

121 S. Dawre, S. Pathak, S. Sharma and P. V. Devarajan, Enhanced antimalalarial activity of a prolonged release in situ gel of arteether-lumefantrine in a murine model, Eur. J. Pharm. Biopharm., 2018, 123, 95-107.

122 W. Peters, Drug resistance in Plasmodium berghei Vincke and Lips, 1948. III. Multiple drug resistance, Exp. Parasitol., 1965, 17, 97-102.

123 R. M. Kannan, E. Nance, S. Kannan and D. A. Tomalia, Emerging concepts in dendrimer-based nanomedicine: from design principles to clinical applications, J. Intern. Med., 2014, 276, 579-617.
124 D. Bhadra, S. Bhadra and N. K. Jain, Pegylated lysine based copolymeric dendritic micelles for solubilization and delivery of artemether, J. Pharm. Pharm. Sci., 2005, 8, 467-482.

125 D. Bhadra, A. K. Yadav, S. Bhadra and N. K. Jain, Glycodendrimeric nanoparticulate carriers of primaquine phosphate for liver targeting, Int. J. Pharm., 2005, 295, 221-233.

126 P. Agrawal, U. Gupta and N. K. Jain, Glycoconjugated peptide dendrimers-based nanoparticulate system for the delivery of chloroquine phosphate, Biomaterials, 2007, 28, 3349-3359.

127 D. Bhadra, S. Bhadra and N. K. Jain, PEGylated peptide dendrimeric carriers for the delivery of antimalarial drug chloroquine phosphate, Pharm. Res., 2006, 23, 623-633.

128 E. M. Coma-Cros, A. Lancelot, M. San Anselmo, L. N. BorghetiCardoso, J. J. Valle-Delgado, J. L. Serrano, X. FernàndezBusquets and T. Sierra, Micelle carriers based on dendritic macromolecules containing bis-MPA and glycine for antimalarial drug delivery, Biomater. Sci., 2019, 7, 1661-1674.

129 J. Movellan, P. Urbán, E. Moles, J. M. de la Fuente, T. Sierra, J. L. Serrano and X. Fernàndez-Busquets, Amphiphilic dendritic derivatives as nanocarriers for the targeted delivery of antimalarial drugs, Biomaterials, 2014, 35, 7940-7950.

130 T. Fröhlich, F. Hahn, L. Belmudes, M. Leidenberger, O. Friedrich, B. Kappes, Y. Couté, M. Marschall and S. B. Tsogoeva, Synthesis of artemisinin-derived dimers, trimers and dendrimers: investigation of their antimalarial and antiviral activities including putative mechanisms of action, Chem. - Eur. J., 2018, 24, 8103-8113.

131 W. J. Youngs, A. R. Knapp, P. O. Wagers and C. A. Tessier, Nanoparticle encapsulated silver carbene complexes and their antimicrobial and anticancer properties: a perspective, Dalton Trans., 2012, 41, 327-336.

132 M. Rai, A. P. Ingle, P. Paralikar, I. Gupta, S. Medici and C. A. Santos, Recent advances in use of silver nanoparticles as antimalarial agents, Int. J. Pharm., 2017, 526, 254-270.

133 G. Benelli, F. Maggi, R. Pavela, K. Murugan, M. Govindarajan, B. Vaseeharan, R. Petrelli, L. Cappellacci, S. Kumar, A. Hofer, M. R. Youssefi, A. A. Alarfaj, J. S. Hwang and A. Higuchi, Mosquito control with green nanopesticides: towards the One Health approach? A review of non-target effects, Environ. Sci. Pollut. Res., 2018, 25, 10184-10206.

134 G. Benelli, Plant-mediated biosynthesis of nanoparticles as an emerging tool against mosquitoes of medical and veterinary importance: a review, Parasitol. Res., 2016, 115, 23-34.

135 G. Benelli and M. Govindarajan, Green-synthesized mosquito oviposition attractants and ovicides: towards a nanoparticle-based "lure and kill" approach?, J. Cluster Sci., 2017, 28, 287-308.

136 C. Panneerselvam, S. Ponarulselvam and K. Murugan, Potential anti-plasmodial activity of synthesized silver nanoparticle using Andrographis paniculata Nees (Acanthaceae), Arch. Appl. Sci. Res., 2011, 3, 208-217.

137 C. Panneerselvam, K. Murugan, M. Roni, A. T. Aziz, U. Suresh, R. Rajaganesh, P. Madhiyazhagan, J. Subramaniam, D. Dinesh, 
M. Nicoletti, A. Higuchi, A. A. Alarfaj, M. A. Munusamy, S. Kumar, N. Desneux and G. Benelli, Fern-synthesized nanoparticles in the fight against malaria: LC/MS analysis of Pteridium aquilinum leaf extract and biosynthesis of silver nanoparticles with high mosquitocidal and antiplasmodial activity, Parasitol. Res., 2016, 115, 997-1013.

138 G. Rajakumar, A. A. Rahuman, I. M. Chung, A. V. Kirthi, S. Marimuthu and K. Anbarasan, Antiplasmodial activity of eco-friendly synthesized palladium nanoparticles using Eclipta prostrata extract against Plasmodium berghei in Swiss albino mice, Parasitol. Res., 2015, 114, 1397-1406.

139 L. Karthik, G. Kumar, T. Keswani, A. Bhattacharyya, B. P. Reddy and K. V. B. Rao, Marine actinobacterial mediated gold nanoparticles synthesis and their antimalarial activity, Nanomedicine, 2013, 9, 951-960.

140 D. Kannan, N. Yadav, S. Ahmad, P. Namdev, S. Bhattacharjee, B. Lochab and S. Singh, Pre-clinical study of iron oxide nanoparticles fortified artesunate for efficient targeting of malarial parasite, EBioMedicine, 2019, 45, 261-277.

141 A. Mehmood, H. Ghafar, S. Yaqoob, U. F. Gohar and B. Ahmad, Mesoporous silica nanoparticles: a review, J. Dev. Drugs, 2017, 6, 1000174.

142 C. Bharti, U. Nagaich, A. K. Pal and N. Gulati, Mesoporous silica nanoparticles in target drug delivery system: a review, Int. J. Pharm. Invest., 2015, 5, 124-133.

143 S. A. Amolegbe, H. Ohmagari, K. Wakata, H. Takehira, R. Ohtani, M. Nakamura, C. Yu and S. Hayami, Synthesis of mesoporous materials as nano-carriers for an antimalarial drug, J. Mater. Chem. B, 2016, 4, 1040-1043.

144 S. A. Amolegbe, Y. Hirano, J. O. Adebayo, O. G. Ademowo, E. A. Balogun, J. A. Obaleye, A. U. Krettli, C. Yu and S. Hayami, Mesoporous silica nanocarriers encapsulated antimalarials with high therapeutic performance, Sci. Rep., 2018, 8, 378.

145 J. S. Beck, J. C. Vartuli, W. J. Roth, M. E. Leonowicz, C. T. Kresge, K. D. Schmitt, C. T. W. Chu, D. H. Olson,
E. W. Sheppard, S. B. McCullen, J. B. Higgins and J. L. Schlenker, A new family of mesoporous molecular sieves prepared with liquid crystal templates, J. Am. Chem. Soc., 1992, 114, 10834-10843.

146 P. Y. Chen, M. Zhang, M. Liu, I. Y. Wong and R. H. Hurt, Ultrastretchable graphene-based molecular barriers for chemical protection, detection and actuation, ACS Nano, 2018, 12, 234-244.

147 C. J. Castilho, D. Li, M. Liu, Y. Liu, H. Gao and R. H. Hurt, Mosquito bite prevention through graphene barrier layers, Proc. Natl. Acad. Sci. U. S. A., 2019, 116, 18304-18309.

148 J. Shi and Y. Fang, Biomedical applications of graphene, in Graphene: Fabrication, Characterizations, Properties and Applications, ed. Z. Hongwei, X. Zhiping, X. Dan, and Y. Fang, Academic Press, Cambridge, MA, USA, 2017, pp. 215-232.

149 Kenry, Y. B. Lim, M. H. Nai, J. Cao, K. P. Loh and C. T. Lim, Graphene oxide inhibits malaria parasite invasion and delays parasitic growth in vitro, Nanoscale, 2017, 9, 14065-14073.

150 S. S. Braga, Cyclodextrins: emerging medicines of the new millennium, Biomolecules, 2019, 9, 801.

151 I. E. Crandall, W. A. Szarek, J. Z. Vlahakis, Y. Xu, R. Vohra, J. Sui and R. Kisilevsky, Sulfated cyclodextrins inhibit the entry of Plasmodium into red blood cells: implications for malarial therapy, Biochem. Pharmacol., 2007, 73, 632-642.

152 A. G. Balducci, E. Magosso, G. Colombo, F. Sonvico, N. A. K. Khan, K. H. Yuen, R. Bettini, P. Colombo and A. Rossi, Agglomerated oral dosage forms of artemisinin/ $\beta$-cyclodextrin spray-dried primary microparticles showing increased dissolution rate and bioavailability, AAPS PharmSciTech, 2013, 14, 911-918.

153 Y. Hao, S. Chen, L. Tian, Z. Yang and G. Huang, Inclusion complex of artemether with 2-hydroxypropyl- $\beta$-cyclodextrin for the treatment of malaria: preparation, characterization and evaluation, MOJ Bioequivalence Bioavailability, 2017, 3, 145-151. 